\title{
On the Formation of SMC X-1: the Effect of Mass and Orbital Angular Momentum Loss
}

\author{
Tao Li and X.-D. Li \\ ${ }^{1}$ Department of Astronomy, Nanjing University, Nanjing 210093, China \\ 2 The Key Laboratory of Modern Astronomy and Astrophysics, Ministry of Education, \\ Nanjing 210093, China \\ litao@nju.edu.cn, lixd@nju.edu.cn
}

\begin{abstract}
SMC X-1 is a high-mass X-ray binary with an orbital period of 3.9 days. The mass of the neutron star is as low as $\sim 1 M_{\odot}$, suggesting that it was likely to be formed through an electron-capture supernova rather an iron-core collapse supernova. From the present system configurations, we argue that the orbital period at the supernova was $\lesssim 10$ days. Since the mass transfer process between the neutron star's progenitor and the companion star before the supernova should have increased the orbital period to tens of days, a mechanism with efficient orbit angular momentum loss and relatively small mass loss is required to account for its current orbital period. We have calculated the evolution of the progenitor binary systems from zero-age main-sequence to the pre-supernova stage with different initial parameters and various mass and angular momentum loss mechanisms. Our results show that the outflow from the outer Langrangian point or a circumbinary disk formed during the mass transfer phase may be qualified for this purpose. We point out that these mechanisms may be popular in binary evolution and significantly affect the formation of compact star binaries.
\end{abstract}

Subject headings: stars: evolution — binaries: close — stars: neutron — pulsars: individual: SMC X-1

\section{Introduction}

High-mass X-ray binaries (HMXBs) usually contain an accreting neutron star (NS) and an early-type $(\mathrm{O}$ or $\mathrm{B})$ companion with mass exceeding $\sim 10 M_{\odot}$. The NS is fed by the stellar wind or beginning Roche-lobe overflow (RLOF) from the companion. Since the 
NSs in HMXBs have experienced very little accretion because of their young ages, their masses should be very close to those at birth. Eclipsing X-ray binary systems where the $\mathrm{X}$-ray source is a pulsar can be ideal systems for a dynamical determination of the NS masses. The measured masses of NSs in HMXBs range from $1.06_{-010}^{+0.11} M_{\odot}$ for SMC X-1 (van der Meer et al. 2007) to 1.86 $\pm 0.16 M_{\odot}$ for Vela X-1 (Barziv et al. 2001; Quaintrell et al. 2003). Recently Rawls et al. (2011) presented an improved method for determining the masses of NSs in eclipsing X-ray pulsars and applied it to six systems. They found the NS masses of $1.77 \pm 0.08 M_{\odot}$ for Vela X-1 and $1.04 \pm 0.09 M_{\odot}$ for SMC X-1. In this paper, we focus on SMC X-1 with a NS mass near the minimum mass limit expected for a NS produced in a supernova (SN) (Haensel et al. 2002; Lattimer \& Prakash 2004).

SMC X-1 was first detected during a rocket flight (Price et al. 1971). The discovery of X-ray eclipses with the Uhuru satellite established the binary nature of SMC X-1. The pulsar has a pulse period of $0.71 \mathrm{~s}$ (Lucke et al. 1976). The companion to the X-ray pulsar, Sk 160, is a B0 I supergiant located in the "wing" of the Small Magellanic Cloud (SMC) at a distance of $60 \mathrm{kpc}$. Its mass was estimated to be $17.2 \pm 0.6 M_{\odot}$ (Revnolds et al. 1993), $16.6 \pm 0.4 M_{\odot}$ (Val Baker et al. 2005), and $15.7_{-1.4}^{+1.5} M_{\odot}$ (van der Meer et al. 2007), while more recent work by Rawls et al. (2011) gives $15.35 \pm 1.53 M_{\odot}$. The X-ray source exhibits an eclipse duration of $0.610 \pm 0.019$ day (Primini et al. 1976) in a 3.892 day orbit. Timing studies of the X-ray pulsations (Levine et al. 1993) give $a_{\mathrm{X}} \sin i=53.4876 \pm 0.0004 \mathrm{ltsec}$ for the projected semi-major axis and indicate a circular orbit with an eccentricity $e<0.00004$. A decay in the orbital period at a rate $\dot{P}_{\text {orb }} / P_{\text {orb }}=(-3.36 \pm 0.02) \times 10^{-6} \mathrm{yr}^{-1}$ was found (Levine et al. 1993), probably due to tidal interaction between the orbit and the rotation of the companion star, which is supposed to be in the hydrogen shell burning phase. The observed period change may also be explained by the self-sustaining mass loss through the outer Lagrangain $\left(L_{2}\right)$ point (Phillips \& Podsiadlowski 2002). The X-ray emission from SMC X-1 has also been found to exhibit a long quasi-stable super-orbital period of 40-60 days, which is believed to be a result of obscuration of the NS by a warped, precessing accretion disk (Wojdowski et al. 1998; Clarkson et al. 2003). In X-rays both low- and high-intensity states have been observed with an X-ray luminosity $L_{\mathrm{X}}$ varying from $\sim 10^{37} \mathrm{ergs}^{-1}$ to $\sim 5 \times 10^{38} \mathrm{ergs}^{-1}$ (Schreier et al. 1972). The mass transfer in SMC X-1 probably has significant contribution from RLOF (van Paradijs \& Kuiper 1984), as the stellar winds from Sk 160 are not strong enough to power the X-rays (Hammerschlag-Hensberge et al. 1984). The X-ray pulsar in the system has a short spin period (seconds) compared to those in wind-fed systems (minutes), as the mass and angular-momentum accretion rate in RLOF systems is much higher than in the latter. Since its discovery, observations with various X-ray telescopes show a steady spin-up of the NS. This makes SMC X-1 an exceptional X-ray pulsar in which no spin-down episode has been observed (Kahabka \& Li 1999). Li \& van den Heuvel (1997) suggested that the 
magnetic moment in SMC X-1 may be as low as that of the bursting pulsar GRO J1744-28, i.e. $\sim 10^{29} \mathrm{Gcm}^{3}$.

Recent analysis by van der Meer et al. (2007) and Rawls et al. (2011), like several earlier investigations, found a low value for the NS mass $\sim 1 M_{\odot}$. Both theories and statistical analyses suggest that the initial masses of NSs might have a bimodal underlying distribution, probably originating from two different formation mechanisms: core collapse supernovae (CCSNe) and electron-capture supernovae (ECSNe) (e.g. Pfahl et al. 2002; van den Heuvel 2004; Podsiadlowski et al. 2004, 2005; Schwab et al. 2010; Kiziltan et al. 2010; Zhang et al. 2011; Özel et al. 2012). A CCSN occurs when a high-mass star develops a degenerate iron core that exceeds the Chandrasekhar limit, while an ECSN is associated with the collapse of a lower-mass ONeMg core as it loses pressure support owing to the sudden capture of electrons by the $\mathrm{Mg}$ and Ne nuclei (Nomoto 1984). Numerical simulations indicate that ECSNe are under-energetic compared to CCSNe by at least an order of magnitude. However they are, at the same time, relatively fast, neutrino-driven, delayed explosions. The short time available for the propagation of the shock is expected to prevent a strong build-up of core asymmetries, leading to typical kicks of $\lesssim 100 \mathrm{kms}^{-1}$, lower than those in CCSN events (Dessart et al. 2006; Kitaura et al. 2006). As ECSNe should produce somewhat less massive NSs $\left(\lesssim 1.3 M_{\odot}\right)$ than CCSNe (Nomoto 1984), the low mass of the NS in SMC X-1 would be consistent with its formation by an ECSN in a degenerate ONeMg core, which is is expected to form at the end of the evolution of stars with masses of $\sim 8-13 M_{\odot}$ (Podsiadlowski et al. 2005); Kitaura et al. 2006). Thus, assuming an explosive mass loss during the formation of the NS of about $1 M_{\odot}$ and a few solar mass of stellar wind mass loss from the NS progenitor, van der Meer et al. (2007) concluded that a $13 M_{\odot}+9 M_{\odot}$ main-sequence progenitor system would be consistent with the present configuration of SMC X-1. The more massive star (i.e., the progenitor of the NS) first evolves to fill its RL, triggering mass transfer to the less massive star. Since the initial mass ratio is not extreme, the binary is unlikely to undergo common envelope (CE) evolution before the SN that cause its orbit to shrink rapidly (for reviews of CE evolution, see Taam \& Sandquist 2000; Ivanova et al. 2013). However, a potential problem with such a scenario is that quasi-conservation of mass and orbital angular momentum during the mass transfer would lead to a fairly wide pre-SN system, such that the present orbital period of $\sim 3.9$ days would be hard to understand, unless a large amount of orbital angular momentum has been lost with relatively small mass (at most a few solar masses) from the system. In this paper, we will try to search plausible evolutionary tracks for SMC X-1, taking into account various kinds of orbital angular momentum loss mechanisms.

This paper is organized as follows. In Section 2 we describe the stellar evolution code and the angular momentum loss mechanisms used. We present the calculated results of the binary evolution in Section 3. Discussion and conclusions are given in Section 4. 


\section{Calculations of Binary Evolution}

\subsection{The Stellar Evolution Code}

We adopt an updated version of the stellar evolution code originally developed by Eggleton (1971, 1972) to compute the binary evolution. The binary system is initially composed of two zero-age main-sequence (ZAMS) stars with an orbital period $P_{\text {orb }}$. We set the more massive star as the primary (of mass $M_{1}$ ), which fills the RL first and is the progenitor of the NS, and the other one as the secondary (of mass $\left.M_{2}\right)$. Low metallicities $(Z=0.004$ and 0.01) are taken for each star according to the environment of the SMC (Piatti 2012). The effective radius $R_{L, 1}$ of the RL for the primary star is calculated from the Eggleton (1983)'s equation,

$$
\frac{R_{L, 1}}{a}=\frac{0.49 q^{2 / 3}}{0.6 q^{2 / 3}+\ln \left(1+q^{1 / 3}\right)},
$$

where $q=M_{1} / M_{2}$ is the mass ratio of the binary components and $a$ is the orbital separation. The rate of mass transfer via RLOF is calculated with $\dot{M}_{1}=\operatorname{RMT} \times \max \left[0,\left(R_{1} / R_{L, 1}-\right.\right.$ $\left.1)^{3}\right] M_{\odot} \mathrm{yr}^{-1}$ in the code, where $R_{1}$ is the radius of the primary, and we adopt $\mathrm{RMT}=10^{3}$ in the calculation.

\subsection{Mass and Angular Momentum Loss Mechanisms}

We assume that the primary star rotates synchronously with the binary orbital revolution, since the timescale of tidal synchronization is generally much shorter than the characteristic evolutionary timescale of the binaries considered here. We consider two kinds of mechanisms of angular momentum loss. The first is the angular momentum loss due to gravitational radiation. This becomes important when the orbital period is short. The rate of the angular momentum loss is given by (Landau \& Lifshitz 1975)

$$
\frac{d J_{\mathrm{GR}}}{d t}=-\frac{32}{5} \frac{G^{7 / 2}}{c^{5}} \frac{M_{1}^{2} M_{2}^{2}\left(M_{1}+M_{2}\right)^{-1 / 2}}{a^{7 / 2}}
$$

where $J, G$, and $c$ are the orbital angular momentum, gravitational constant, and speed of light, respectively. The second and more important angular momentum loss mechanism is nonconservative mass transfer. During the mass-transfer processes, part of the transferred mass from the primary star may escape the binary system, carrying away the orbital angular momentum (Warner 1978; Eggleton 2000). This mass loss might be related to the rapid rotation of the accreting star. Since the accreting matter carries a certain angular momentum that will be transferred to the secondary (Packet 1981), this angular momentum spins up the top layers of the secondary star, and is transferred further into the star due to rotationally 
induced mixing processes. When the secondary is spun up to close to critical rotation it starts losing mass due to the influence of centrifugal force (e.g. Langer 1998), although it is not clear in which ways the material leaves the binary. Considering the complicated processes of mass loss, we adopt four kinds of mass loss as follows. In the first case we assume that a fraction $\alpha$ of the transferred mass is ejected out of the binary as isotropic winds from the secondary, carrying away the specific angular momentum of the secondary,

$$
\frac{d J_{\mathrm{ml}, 1}}{d t}=-\alpha \dot{M}_{1}\left(\frac{q}{1+q}\right)^{2} a^{2} \omega
$$

where $\omega$ is the angular velocity of the binary. Alternatively, the transferred mass might be lost at the inner Lagrangian $\left(L_{1}\right)$ point and the corresponding rate of angular momentum loss is

$$
\frac{d J_{\mathrm{ml}, 2}}{d t}=-\beta \dot{M}_{1} a_{L 1}^{2} \omega
$$

where $\beta$ is the fraction of mass loss, and $a_{L 1}$ is the distance from the $L_{1}$ point to the center of mass of the binary system.

In some cases, the matter surrounding the two components may expand to the outer Lagrangian $\left(L_{2}\right)$ point, and part of the material lost from the donor star may escape the system through the $L_{2}$ point (Drechsel et al. 1995; Vanbeveren et al. 1998). Assuming that a fraction $\epsilon$ of the matter flow leaves the binary, the angular momentum loss rate due to the $L_{2}$ point outflow is given by

$$
\frac{d J_{\mathrm{ml}, 3}}{d t}=-\epsilon \dot{M}_{1} a_{L 2}^{2} \omega
$$

where $a_{L 2}$ is the distance between the mass center of binary and the $L_{2}$ point. In our calculation, we take into account the $L_{2}$ point outflow after the mass ratio inverts, to ensure that the outflows is on the side of the lower-mass star that fills the RL.

The last case is the angular momentum loss due to a circumbinary (CB) disk. CB disks were first proposed by van den Heuvel \& De Loore (1973) when investigating the evolution of X-ray binaries. Recently, CB disks are considered in a wide variety of astrophysical objects, e.g., young binary stars, protoplanetary systems, cataclysmic variables (CVs) and massive binary black hole systems in active galactic nuclei (Spruit \& Taam 2001; Hayasaki \& Okazaki 2009; Shao \& Li 2012, and references therein). It has been argued that during mass exchange in binary systems, some of the lost matter which possesses high orbital angular momentum may form a disk surrounding the binary system rather than leave the binary system (van den Heuvel 1994). As shown by Spruit \& Taam (2001) and Chen \& Li (2006), CB disks can efficiently extract orbit angular momentum from the binary, and enhance the mass transfer rates. In this work, we assume that a fraction $\delta$ of the mass lost from the donor feeds into the CB disk. At the inner edge $r_{\mathrm{i}}$ of the disk tidal torques are then exerted 
on the binary via gravitational interaction, and the corresponding angular momentum loss rate is (Spruit \& Taam 2001)

$$
\frac{d J_{\mathrm{CB}}}{d t}=-\gamma\left(\frac{2 \pi a^{2}}{P_{\mathrm{orb}}}\right) \dot{M}_{\mathrm{CB}}\left(\frac{t}{t_{\mathrm{vi}}}\right)^{1 / 3}
$$

where $\dot{M}_{\mathrm{CB}}=\delta \dot{M}_{1}, \gamma^{2}=r_{\mathrm{i}} / a=1.7$ (Artymowicz \& Lubow 1994),$t$ is the time since the onset of the $\mathrm{CB}$ disk formation, and $t_{\mathrm{vi}}$ is the viscous timescale at the inner edge of the $\mathrm{CB}$ disk. For the standard $\alpha$-viscosity disk (Shakura \& Sunvaev 1973),

$$
t_{\mathrm{vi}}=\frac{2 \gamma^{3} P_{\mathrm{orb}}}{3 \pi \alpha_{\mathrm{SS}}\left(H_{\mathrm{i}} / r_{\mathrm{i}}\right)^{2}},
$$

where $H_{\mathrm{i}}$ is the scale height of the disk at the inner edge. In the following calculation we set the viscosity parameter $\alpha_{\mathrm{SS}}=0.01$, and assume that the disk is hydrostatically supported, and geometrically thin with $H_{\mathrm{i}} / r_{\mathrm{i}} \sim 0.03$ (Belle et al. 2004).

\section{The Formation of SMC X-1}

The formation of a HMXB like SMC X-1 requires two initially massive stars. When it evolves to become a supergiant and fill its RL, the primary star transfers material to the secondary on a thermal timescale, and the binary orbit shrinks rapidly at first. Because the mass ratio is not far from unity, the mass transfer process is dynamically stable without the occurrence of a CE. A large fraction of the transferred matter is expected to be accreted by the secondary star. When the mass ratio inverts, further mass transfer leads to increase of the orbital period. Finally the primary star develops an ONeMg core, which collapses to be a NS. At this time the primary star is much less massive than the secondary. Considering the fact that a small fraction of the binary mass is lost and a small kick is imparted to the newborn NS during the SN, the binary orbit should be nearly circular or mildly eccentric after the SN. During the subsequent evolution, since stellar winds of the main-sequence secondary is not strong, there is hardly any mass transfer until the secondary starts to fill its RL. So we expect that the masses of the NS and the optical companion has changed little since the SN, and will use them to constrain the binary parameters before the SN. It is noted that, after the onset of the secondary RLOF, the large mass ratio between the companion star and the NS will cause the orbit to shrink in response to mass transfer, most likely leading to a $\mathrm{CE}$ event. 


\subsection{The effect of the SN kick}

When a star explodes as a SN, the NS formed receives a velocity kick due to any asymmetry in the explosion (Lvne \& Lorimer 1994). This also imparts an impulse to the companion star resulting in an eccentric binary orbit. As mentioned earlier, the low mass of the NS in SMC X-1 suggests that its formation is likely to be due to an electron-capture collapse with a low kick velocity, which results in a small eccentricity. Tidal interaction between the NS and the companion star can also circularize the binary orbit if it is narrow enough.

We first try to estimate the possible distributions of the orbital period before the SN and the eccentricity after the SN from current binary parameters. The NS mass $\left(\sim 1 M_{\odot}\right)$, the secondary mass $\left(\sim 16 M_{\odot}\right)$ and the orbital period $(\sim 4 \mathrm{~d})$ have already been estimated from the observations of SMC X-1. The mass of the secondary is assumed to be constant during the SN, and the primary's mass before the SN can be constrained by the range of the helium core mass $\left(\sim 1.37 M_{\odot}-2.5 M_{\odot}\right)$ that leads to an ECSN (Nomoto 1984; Podsiadlowski et al. 2004). The $\mathrm{SN}$ is thought to take place in a circular orbit because of the previous mass transfer, and the kick direction is assumed to be uniform over all directions. We take the kick velocity $v_{\mathrm{k}}$ from a Maxwellian distribution

$$
P\left(v_{\mathrm{k}}\right)=\sqrt{\frac{2}{\pi}} \frac{v_{\mathrm{k}}^{2}}{\sigma_{\mathrm{k}}^{3}} \mathrm{e}^{-v_{\mathrm{k}}^{2} / 2 \sigma_{\mathrm{k}}^{3}},
$$

where the velocity dispersion $\sigma_{\mathrm{k}}$ is usually taken to be $190 \mathrm{kms}^{-1}$ (Hansen \& Phinney 1997) or $265 \mathrm{kms}^{-1}$ (Hobbs et al. 2005) based on the analysis of various pulsar proper motion samples for CCSN events. The kick velocities must be much lower for ECSNe (e.g., Pfahl et al. 2002). A small kick value ( $10 \%$ of the standard NS kick, i.e., $\sigma_{\mathrm{k}}=26.5 \mathrm{kms}^{-1}$ ) was adopted by Linden et al. (2009), in order to estimate the overconcentration of ECSN sources in the SMC bar. Here we set $\sigma_{\mathrm{k}}=20,50$, and $70 \mathrm{kms}^{-1}$ to examine the effect of ECSN kicks. Figure 1 shows the derived distributions of the pre-SN orbital period (top panel) and the post-SN eccentricity (middle panel) for a $2 M_{\odot}+16 M_{\odot}$ pre-SN system, with the assumption that the post-SN period is $\sim 4$ days. The results with the CCSN kicks are also presented for comparison. With the increase of $\sigma_{\mathrm{k}}$, a more extended range of the pre-SN orbital period can result in the 4 day post-SN period, and the post-SN eccentricity tends to be larger. For sufficiently large kicks the eccentricity can be larger than unity so that the binary is disrupted. For CCSNe this occurs at the high-end in the distribution of the pre-SN period, providing an upper limit of $\sim 12$ days. This means that a $>12$ day pre-SN binary would not evolve to a 4 day post-SN binary with the SMC X-1 mass parameters. From the contours in the orbital period vs. eccentricity plane (the bottom panel), one can see the trend of pre-SN

period and post-SN eccentricity distributions. There are two "wings" with the center close 
to the post-SN period. The most probable regions vary from the center to the wings when $\sigma_{\mathrm{k}}$ is increasing. For CCSN kicks, to form the expected post-SN systems like SMC X-1 requires a pre-SN period $\lesssim 2$ days, and the post-SN eccentricity is high $(\gtrsim 0.4)$. For ECSNe a lower eccentricity is expected and a relatively longer pre-SN period $(\sim 2 \mathrm{~d}-8 \mathrm{~d})$ is required.

\subsection{The Pre-SN Binary Evolution}

Following the above analysis, we simplify the formation of SMC X-1 to be the evolution of a ZAMS binary system to a pre-SN binary with the orbital period $\sim 2 \mathrm{~d}-8 \mathrm{~d}$, the primary (He star)'s mass $\sim 2 M_{\odot}$, and the secondary's mass $\sim 15-18 M_{\odot}$. We select two sets of mass parameters for the progenitor binary to illustrate the possible evolutionary tracks of SMC $\mathrm{X}-1$. They are $9.98 M_{\odot}+8.02 M_{\odot}$ and $12.56 M_{\odot}+5.60 M_{\odot}$ ZAMS binaries with similar initial total masses for the conservative evolution. We adjust the initial mass of the secondary if the evolutionary model is nonconservative, in order to keep the secondary mass at the SN within the suitable range.

\subsubsection{The binary evolution with $M_{1, \mathrm{i}}=9.98 M_{\odot}$}

We first consider the evolution of the progenitor system with the initial primary's mass $M_{1, \mathrm{i}}=9.98 M_{\odot}$. To examine the possible evolutionary tracks and the influence of different angular momentum loss mechanisms, we construct five models with various kinds of mass and angular momentum loss mentioned in Section 2.2: (1) conservative mass transfer; (2) nonconservative mass transfer with mass loss from the secondary; (3) nonconservative mass transfer with mass loss from the $L_{1}$ point; (4) nonconservative mass transfer with mass loss from the $L_{2}$ point; and (5) nonconservative mass transfer with a small fractional mass feeding into the $\mathrm{CB}$ disk. The initial orbit periods are taken to be $P_{\text {orb,i }}=1.5,2.0,2.5,3.0,3.5,4.0$, 5.0, 7.0, and 10.0 days. At the smallest orbital period the zero-age primary star just fits the size of the RL. The metallicities are adopted to be $Z=0.01$ and 0.004 .

The results of the pre-SN evolution for different initial orbital periods are summarized in Tables 1 (for models [1]-[4]), 2, and 3 (for model [5]). In the tables $M_{1, \mathrm{f}}, M_{2, \mathrm{f}}$, and $P_{\text {orb,f }}$ are the primary (He star)'s mass, the secondary's mass and the orbital period close to the end of the primary's evolution, respectively. In Table 1 we distinguish Cases A and B mass transfer, which occurs when the primary burns hydrogen in its core and evolves off the main-sequence but before helium core ignition, respectively. The Case B mass transfer is most likely to be unstable if the value of $\epsilon$ is high for model (4). This is what " $\dot{M}$ divergent" means in the 
tables. So we only present the results of model (2)-(4) with Case A and stable Case B mass transfer.

The results of the first three models in Table 1 show little distinction. The orbital angular momentum loss from the binaries is all inefficient, so that the final orbital periods are usually larger than 40 days (for $Z=0.01$ ) or 25 days (for $Z=0.004$ ), inconsistent with those for SMC X-1. It is also noted that a larger $Z$ leads to longer $P_{\text {orb,f }}$ because of longer mass transfer time. For each model, the higher $P_{\text {orb,i }}$, the heavier the evolved He star and the lighter the secondary. It is interesting to note that $P_{\text {orb,f }}$ decreases with increasing $P_{\text {orb,i }}$ for Case A mass transfer, but increases with $P_{\text {orb, } \mathrm{i}}$ for Case $\mathrm{B}$ mass transfer. This means the lowest pre-SN period $P_{\text {orb,f }}$ corresponds to the $P_{\text {orb,i }}$ around the boundary between Case A and Case B evolution. Different from the first three models, in model (4) the mass loss from the $L_{2}$ point can cause secular orbital shrinking. If the mass loss fraction $\epsilon \sim 25 \%, P_{\text {orb,f }}$ becomes shorter than 10 days, which satisfies what is needed for the formation of SMC X-1.

Although the $L_{2}$ point outflow provides a way for the formation of relatively compact systems. this mechanism requires a considerable mass loss rate, and sometimes the primary's mass at the end of the evolution is lower than the range for an ECSN. Thus in model (5) we take into account the influence of a CB disk formed during the mass transfer. In this case we adopt the progenitor binary system of $9.98 M_{\odot}+8.12 M_{\odot}$ with the secondary's mass slightly higher than that in model (1), and we only calculate the evolution with $P_{\text {orb,i }}$ near the the boundary between Case A and Case B mass transfer. Similar as in model (4), the Case B mass transfer becomes unstable if $\delta$ is large, so we mainly focus on Case A evolution. The results are presented in Tables 2 and 3 with $Z=0.01$ and $Z=0.004$, respectively. We can always find that it is able to form a pre-SN binary system with a relatively small $\delta$ (a few hundredth), which meets the SMC X-1 requirements. The wider the initial system, the higher $\delta$ needed.

We then select some representative scenarios from the tables to demonstrate the detailed evolution of the binary. Figure 2 shows the pre-SN evolutionary tracks of the primary of initial mass $9.98 M_{\odot}$ in the $\mathrm{H}-\mathrm{R}$ diagram. The panels from top to bottom correspond to models (1)-(5), respectively. In the left, middle and right columns the initial orbital period $P_{\text {orb,i }}$ is taken to be 2.0, 2.5, and 3.0 days, respectively. For model (1), the initial secondary's mass is $M_{2, \mathrm{i}}=8.02 M_{\odot}$; for models (2)-(4), we adopt $\alpha=\beta=\epsilon=0.25$; for model (5), $M_{2, \mathrm{i}}=9.62 M_{\odot}$, and $M_{2, \mathrm{i}}=8.12 M_{\odot}$. The solid and dotted lines are obtained with $Z=0.01$ and 0.004 , respectively. In Fig. 3, we compare the mass transfer rates between different models with different $P_{\text {orb }, \mathrm{i}}$. The figure is organized same as Fig. 2. The only Case B evolution is for $P_{\text {orb,i }}=3.0$ days and $Z=0.004$, and in other scenarios are all Case A evolutions. We can see that there are not any significant differences in the evolutionary tracks and in the 
mass transfer rates among the first three models with the same $P_{\text {orb,i }}$, while the other two models are distinct from the others. The binaries generally experience three (or two, the first two phases are sometimes degenerated) phases of mass transfer in Case A evolution in Fig. 3. In the first phase the mass transfer proceeds on a thermal timescale (see Langer et al. 2000, for further discussions on thermally unstable mass transfer). The outcome in this situation is that the donor star overfills its RL even more, leading to further mass loss. Although the star is driven out of thermal equilibrium during this phase, it manages to retain hydrostatic equilibrium and the system can in this case avoid a so-called delayed dynamical instability (Hjellming \& Webbink 1987; Kalogera \& Webbink 1996), which would have resulted in a CE stage. The second and third phases proceed on nuclear timescales determined by the core burning of the remaining hydrogen and, later on, the hydrogen shell burning, respectively. Obviously the wider the initial system, the later the RLOF mass transfer begins, because there is no effective angular momentum loss mechanism before the onset of mass transfer, and the mass transfer timescale becomes shorter. When $P_{\text {orb, } i}$ is so long that there is Case $\mathrm{B}$ mass transfer, the three phases merge into one. Furthermore, lower metallicity causes later onset of RLOF and shorter mass transfer time due to a larger initial stellar radius and ending the red giant stage earlier. For models (4) and (5), if $\epsilon$ or $\delta$ is high enough, the mass transfer can be dynamically unstable, so that the binary enters the CE evolution when the orbital period is as low as $\sim 2$ days.

The orbital period evolution is shown in Fig. 4, which is also organized similar as Fig. 2, but in each subgraph we add the cases with different values of the mass loss fraction in different colored lines for comparison. The dashed horizontal lines indicate the possible range of the pre-SN periods expected to lead to the formation of SMC X-1. It is clearly seen that only in model (4) or (5) with suitable values of $\epsilon$ or $\delta$ can the binary evolve into the proper orbital period.

\subsubsection{The binary evolution with $M_{1, \mathrm{i}}=12.56 M_{\odot}$}

In this subsection we investigate the evolution of the possible progenitor of SMC X-1 with a $12.56 M_{\odot}$ main-sequence primary. Tables 4,5 and 6 present the results of the binary parameters for the primary evolving from ZAMS to pre-SN in different models, similar as those for the $9.98 M_{\odot}$ primary. The orbital period evolution is shown in Fig. 5. Compared with the case of $M_{1, \mathrm{i}}=9.98 M_{\odot}$, the higher mass ratio for $M_{1, \mathrm{i}}=12.56 M_{\odot}$ leads to shorter $P_{\text {orb,f }}$ for similar total mass, initial orbital period and metallicity. Usually models (4) and (5) are more preferred, while in the case of conservative mass transfer, the $P_{\text {orb,f }}$ sometimes nearly fits in the pre-SN period range for the formation of SMC X-1, but the He star mass 
exceeds the upper limit of the He core mass for an ECSN.

Combining the calculated results of binary evolutions with different initial parameters, we can roughly constrain the mass of the NS progenitor in SMC X-1 to be $\sim 10-12.5 M_{\odot}$.

\section{Discussion and Conclusions}

Since the pioneering work of van den Heuvel \& Heise (1972) the formation and evolution of HMXBs with NS components has been intensively studied (Rappaport \& van den Heuvel 1982; Meurs \& van den Heuvel 1989; Pols et al. 1991; Lipunov et al. 1994; Dalton \& Sarazin 1995; Iben. Tutukov, \& Yungelson 1995; Terman. Taam, \& Savage 1998; Lü. Zhu, \& Wang. 2011). The generally accepted evolutionary picture is that, the originally more massive star becomes the less massive as a result of mass transfer or mass loss, leaving behind a system composed of an evolved core and a MS companion. The subsequent evolution of the core to the SN leads to the formation of a NS/massive star binary system. However, there are big uncertainties associated with the effects of mass and angular momentum loss on the evolution of these systems (in the context of the mass transfer and CE evolution). Detailed investigations on the formation of individual HMXBs are also lacking because it is difficult to constrain the initial binary parameters from current observations. The low mass of the NS in SMC X-1 is suggestive of its formation through an ECSN, which provides useful information on the properties of the NS progenitor and the formation scenarios. ECSNe are thought to occur in stars of mass $\sim 8-13 M_{\odot}$. Combining with the total mass of the current binary, this suggests that the initial masses of the primary and secondary stars are comparable so that $\mathrm{CE}$ evolution is not likely to occur, and the orbital evolution is mainly dominated by the mass transfer/loss processes and tidal interaction. If the mass transfer is conservative before the $\mathrm{SN}$, the ratio between the final and initial orbital periods is

$$
\frac{P_{\text {orb }, \mathrm{f}}}{P_{\text {orb }, \mathrm{i}}}=\left(\frac{M_{1, \mathrm{i}} M_{2, i}}{M_{1, \mathrm{f}} M_{2, f}}\right)^{3} \sim 50-100 .
$$

This gives an orbital period at least tens of days when the SN occurs (the orbit may shrink to some extent due to the wind from the primary), and in the further evolution it would be difficult for the orbital period to decrease to $\sim 4$ days by tidal torques within the lifetime of the secondary star. This point has already been notified by Raguzove \& Lipunov (1998) to explain why Be/X-ray binaries are in wide (> 10 days) and eccentric orbits. For SMC X-1, as the orbital change during the post-SN evolution is limited, through numerical calculation of the change in the orbital period during the $\mathrm{SN}$, we simplify the evolutional problem to evolving a progenitor ZAMS binary system up to the pre-SN epoch, when it has developed an $\mathrm{ONeMg}$ core approximately fitting the range expected to lead to an ECSN, with the 
orbital period $\lesssim 8-10$ days, and the secondary mass $\sim 15-17 M_{\odot}$. Obviously these requirements rule out the conservative mass transfer scenario, and the key problem is to find an effective orbital angular loss mechanism with relatively small mass loss to cause secular orbital shrinking. According to our calculation, it could be either mass loss through the $L_{2}$ point or or a $\mathrm{CB}$ disk (note that the formation of a $\mathrm{CB}$ disk might also originate from the outflow from the $L_{2}$ point). The difference is that, in the former case some of the material leaves the system carrying away the orbital angular momentum, while in the latter the lost mass accumulates in a disk surrounding the binary, draining the orbital angular momentum from the binary through tidal torques. Because of the difference in the efficiency of angular momentum loss, the fraction of lost mass in the total transferred mass is significantly higher for the $L_{2}$ point outflow than for the CB disk. Our calculated results indicate that the evolution with the $L_{2}$ point mass loss or a CB disk could lead to the formation of SMC X-1 through Case A mass transfer $\left(P_{\text {orb,i }} \lesssim 3.5\right.$ days $)$ with $M_{1, \mathrm{i}} \sim 10-12.5 M_{\odot}$.

Although our analysis focuses on the formation of SMC X-1, the conclusion that mass transfer is likely to be associated with significant angular momentum loss may be a general feature in the evolution of various kinds of binaries (e.g., Refsdal et al. 1974; Massevitch \& Yungelson 1975; Sarna 1993; Eggleton 2000; Petrovic et al. 2005; van Rensbergen et al. 2008). Previous investigations emphasized the necessity of nonconservative mass transfer to account for the properties of the related binaries. However, the ways of mass loss and the corresponding efficiency of angular momentum loss have not been well constrained. In this work we show that the outflow from the $L_{2}$ point or a circumbinary disk is more preferred than mass loss from the accreting star or the $L_{1}$ point, at least for the formation of SMC X-1.

Similar suggestions can be found in the literature. For example, in order to address the observed lower mass limit $\left(\sim 8 M_{\odot}\right)$ for the Be stars in Be/X-ray binaries, Portegies Zwart (1995) proposed that there may be mass loss from the binary systems at the $L_{2}$ point when the primary star transfers mass to the secondary (who's rotation will be accelerated such that it will become a Be star). The escaped matter takes away about six times the specific angular momentum of the binary system, so that those with small initial mass ratios would undergo spiral-in and evolve towards a CE phase. In the other extreme of low-mass binaries, Knigge et al. (2011) recently reconstructed the complete evolutionary path followed by cataclysmic variables $(\mathrm{CVs})$, using the observed mass-radius relationship of the secondary stars. The best-fit revised model of $\mathrm{CV}$ evolution indicates that the angular momentum loss rate below the period gap is $2.47( \pm 0.22)$ times the rate induced by gravitational radiation, suggesting the existence of some other angular momentum loss mechanisms. Shao \& Li (2012) considered several kinds of consequential angular momentum loss mechanisms including isotropic wind from the accreting white dwarfs, outflows from the Langrangian points, and the formation of a CB disk. They showed that neither isotropic wind from the white 
dwarf nor outflow from the $L_{1}$ point can explain the extra angular momentum loss rate, while outflow from the $L_{2}$ point or a CB disk can satisfy the extra angular momentum loss

provided that $\sim(15-45) \%$ of the transferred mass is lost from the binary, or $\lesssim 10^{-3}$ of the transferred mass goes into the CB disk (see also Spruit \& Taam 2001). These results are in general line with what we have obtained for SMC X-1.

Mass transfer critically affects the evolutionary paths of binaries. Depending on whether it is stable or not, a binary may either survive the initial mass transfer phase to become a semi-detached system or end up merging completely. The stability of mass transfer depends sensitively on the angular momentum transport mechanisms. Including the mass loss from the $L_{2}$ point (or a CB disk) can significantly destabilize the mass transfer processes, enhance the merging rate, and influence the birth rate of compact star binaries. Obviously a thorough investigation on this subject is needed before it can be implemented into future population synthesis calculation.

This work was supported by the Natural Science Foundation of China under grant numbers 11133001 and 11333004, the National Basic Research Program of China (973 Program 2009CB824800), and the Qinglan project of Jiangsu Province.

\section{REFERENCES}

Artymowicz, P., \& Lubow, S. H. 1994, ApJ, 421, 651

Barziv, O., Kaper, L., Van Kerkwijk, M. H., Telting, J. H., \& Van Paradijs, J. 2001, A\&A, 377,925

Belle, K. E., Sanghi, N., Howell, S. B., Holberg, J. B., \& Williams, P. T. 2004, AJ, 128, 448

Chen, W.-C. \& Li, X.-D. 2006, MNRAS, 373, 305

Clarkson, W. I., Charles, P. A., Coe, M. J., et al. 2003, MNRAS, 339, 447

Dessart, L., Burrows, A., Ott, C. D., et al. 2006, ApJ, 644, 1063

Dalton, W. W. \& Sarazin, C. L., 1995, ApJ, 440, 280

Drechsel, H., Haas, S., Lorenz, R., \& Gayler, S. 1995, A\&A, 294, 723

Eggleton, P. P. 1971, MNRAS, 151, 351

Eggleton, P. P. 1972, MNRAS, 156, 361 
Eggleton, P. P. 1983, ApJ, 268, 368

Eggleton, P. 2000, New A Rev., 44, 111

Haensel, P., Zdunik, J. L., \& Douchin, F. 2002, A\&A, 385, 301

Hammerschlag-Hensberge, G., Kallman, T. R., \& Howarth, I. D. 1984, ApJ, 283, 249

Han, Z., Podsiadlowski, P., \& Eggleton, P. P. 1994, MNRAS, 270, 121

Hansen, B. M. S., \& Phinney, E. S. 1997, MNRAS, 291, 569

Hayasaki, K., \& Okazaki, A. T. 2009, ApJ, 691, L5

Hilditch, R. W., Howarth, I. D., \& Harries, T. J. 2005, MNRAS, 357, 304

Hjellming, M. S., \& Webbink, R. F. 1987, ApJ, 318, 794

Hobbs, G., Lorimer, D. R., Lyne, A. G., \& Kramer, M. 2005, MNRAS, 360, 974

Iben, I., Jr, Tutukov, A. V., \& Yungelson, L. R ., 1995, ApJS, 100, 217

Ivanova, N., Justham, S., Chen, X., et al. 2013, A\&A Rev., 21, 59

Kahabka, P., \& Li, X.-D. 1999, A\&A, 345, 117

Kalogera, V., \& Webbink, R. F. 1996, ApJ, 458, 301

Kitaura, F. S., Janka, H.-T., \& Hillebrandt, W. 2006, A\&A, 450, 345

Kiziltan, B., Kottas, A., \& Thorsett, S. E. 2010, arXiv:1011.4291

Knigge, C., Baraffe, I., \& Patterson, J. 2011, ApJS, 194, 28

Landau, L. D., \& Lifshitz, E. M. 1975, Course of theoretical physics - Pergamon International Library of Science, Technology, Engineering and Social Studies, Oxford: Pergamon Press, 1975, 4th rev.engl.ed.

Langer, N. 1998, A\&A, 329, 591

Langer, N., Deutschmann, A., Wellstein, S., Hőflich, P. 2000, A\&A, 362, 1046

Lattimer, J. M., \& Prakash, M. 2004, Science, 304, 536

Levine, A., Rappaport, S., Deeter, J. E., Boynton, P. E., \& Nagase, F. 1993, ApJ, 410, 328

Li, X.-D., \& van den Heuvel, E. P. J. 1997, A\&A, 322, L9 
Liller, W. 1973, ApJ, 184, L37

Linden, T., Sepinsky, J. F., Kalogera, V., \& Belczynski, K. 2009, ApJ, 699, 1573

Lipunov, V. M., Postnov, K. A., Prokhorov, M. E., \& Osminkin, E. Yu. 1994, ApJ, 423, L121

Lü, G., Zhu, C., \& Wang, Z. 2011, RAA, 11, 327

Lucke, R., Yentis, D., Friedman, H., Fritz, G., \& Shulman, S. 1976, ApJ, 206, L25

Lyne, A. G., \& Lorimer, D. R. 1994, Nature, 369, 127

Massevitch, A. \& Yungelson, L. 1975, MmSAI, 46, 217

Meurs, E. J. \& van den Heuvel, E. P. J., 1989, A\&A, 226, 88

Nieuwenhuijzen, H., \& de Jager, C. 1990, A\&A, 231, 134

Nomoto, K. 1984, ApJ, 277, 791

Özel, F., Psaltis, D., Narayan, R., \& Santos Villarreal, A. 2012, ApJ, 757, 55

Packet, W. 1981, A\&A, 102, 17

Petrovic, J., Langer, N., \& van der Hucht, K. A. 2005, A\&A, 435, 1013

Pfahl, E., Rappaport, S., Podsiadlowski, P., \& Spruit, H. 2002, ApJ, 574, 364

Phillips, S. N., \& Podsiadlowski, P. 2002, MNRAS, 337, 431

Piatti, A. E. 2012, MNRAS, 422, 1109

Podsiadlowski, P., Dewi, J. D. M., Lesaffre, P., et al. 2005, MNRAS, 361, 1243

Podsiadlowski, P., Langer, N., Poelarends, A. J. T., et al. 2004, ApJ, 612, 1044

Pols, O. R., Cote, J. Waters, L. B. F. M., \& Heise, J. 1991, A\&A, 241, 419

Pols, O. R., Tout, C. A., Eggleton, P. P., \& Han, Z. 1995, MNRAS, 274, 964

Portegies Zwart, S. F., 1995, A\&A, 296, 691

Price, R. E., Groves, D. J., Rodrigues, R. M., et al. 1971, ApJ, 168, L7

Primini, F., Clark, G. W., Lewin, W., et al. 1976, ApJ, 210, L71 
Quaintrell, H., Norton, A. J., Ash, T. D. C., et al. 2003, A\&A, 401, 313

Raguzove, N. V. \& Lipunov, V. M. 1998, A\&A, 340, 85

Rappaport, S. \& van den Heuvel, E. P. J. 1982, in The Be Stars, ed. M. Jaschek \& H. Groth (Dordrecht: Reidel), 327

Rawls, M. L., Orosz, J. A., McClintock, J. E., et al. 2011, ApJ, 730, 25

Reynolds, A. P., Hilditch, R. W., Bell, S. A., \& Hill, G. 1993, MNRAS, 261, 337

Refsdal, S., Roth, M., \& Weigert, A. 1974, A\&A, 36, 113

Sarna, M. 1993, MNRAS, 262, 534

Schreier, E., Giacconi, R., Gursky, H., Kellogg, E., \& Tananbaum, H. 1972, ApJ, 178, L71

Schwab, J., Podsiadlowski, P., \& Rappaport, S. 2010, ApJ, 719, 722

Shakura, N. I. \& Sunyaev, R. A., 1973, A\&A, 24, 337

Shao, Y. \& Li, X.-D. 2012, ApJ, 745, 165

Spruit, H. C., \& Taam, R. E. 2001, ApJ, 548, 900

Taam, R. E., \& Sandquist, E. L. 2000, ARA\&A, 38, 113

Terman, J. L., Taam, R. E., \& Savage, C. O. 1998, MNRAS, 293, 113

Val Baker, A. K. F., Norton, A. J., \& Quaintrell, H. 2005, A\&A, 441, 685

Vanbeveren, D., De Loore, C., \& Van Rensbergen, W. 1998, A\&A Rev., 9, 63

van den Heuvel, E. P. J. 1994, Saas-Fee Advanced Course 22: Interacting Binaries, 263

van den Heuvel, E. P. J. 2004, 5th INTEGRAL Workshop on the INTEGRAL Universe, 552, 185

van den Heuvel, E. P. J., \& De Loore, C. 1973, A\&A, 25, 387

van den Heuvel, E. P. J. \& Heise, J., 1972, Nature Phys. Sci., 239, 67

van der Meer, A., Kaper, L., van Kerkwijk, M. H., Heemskerk, M. H. M., \& van den Heuvel, E. P. J. 2007, A\&A, 473, 523

van Paradijs, J., \& Kuiper, L. 1984, A\&A, 138, 71 
van Rensbergen, W., De Greve, J. P., De Loore, C., \& Mennekens, N. 2008, A\&A, 487, 1129

Warner, B. 1978, A\&A, 28, 303

Wellstein, S., Langer, N., \& Braun, H. 2001, A\&A, 369, 939

Wojdowski, P., Clark, G. W., Levine, A. M., Woo, J. W., \& Zhang, S. N. 1998, ApJ, 502, 253

Zhang, C. M., Wang, J., Zhao, Y. H., et al. 2011, A\&A, 527, A83 

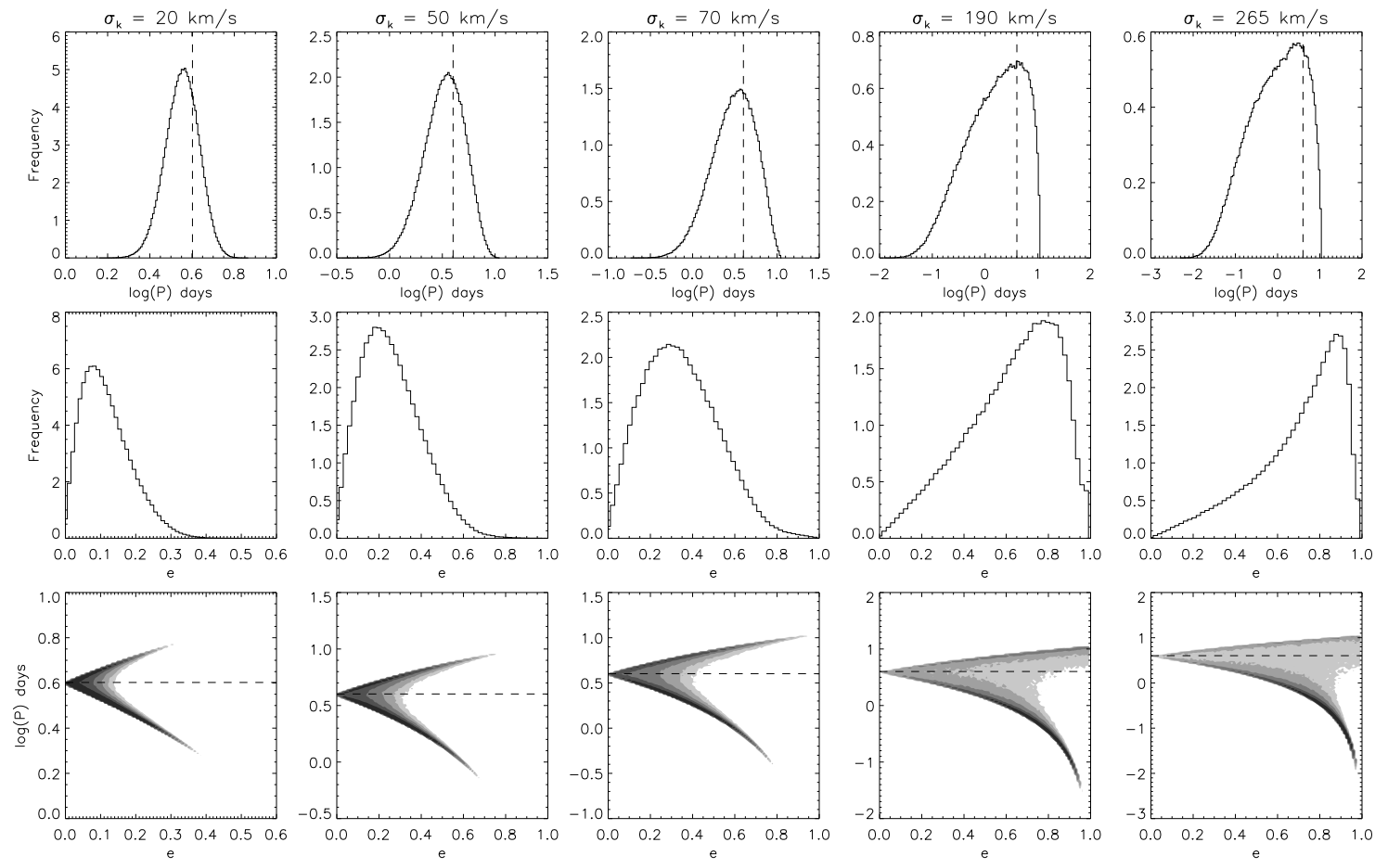

Fig. 1.- Distributions of the pre-SN orbital period (top) and the post-SN eccentricity (middle) for a binary consisting of a $2 M_{\odot}$ He star and a $16 M_{\odot}$ MS star with $\sigma_{\mathrm{k}}=$ $20,50,70,190,265 \mathrm{kms}^{-1}$. The mass of the newborn NS is assumed to be $1 M_{\odot}$. In the bottom panels are the contours representing the relative probabilities of the system parameters in the pre-SN orbital period vs. the post-SN eccentricity plane. The darker the color, the greater the probabilities. The expected post-SN period ( $\sim 4$ days $)$ is also drawn with the dashed line. 


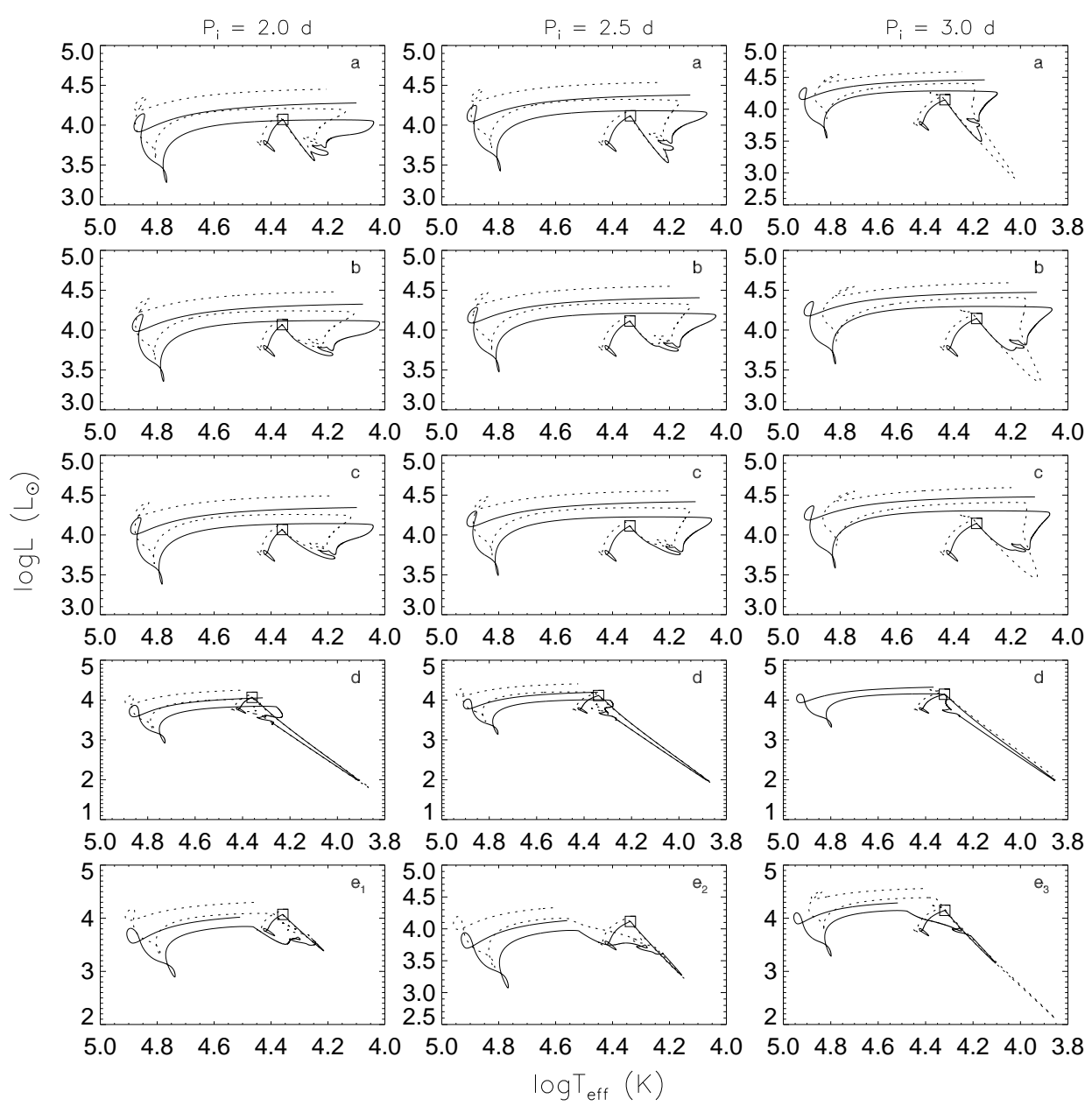

Fig. 2.- The evolutionary tracks for the primary star of mass $9.98 M_{\odot}$ from ZAMS to the formation of the $\mathrm{O}-\mathrm{Ne}-\mathrm{Mg}$ star in the $\mathrm{H}-\mathrm{R}$ diagram. The initial orbital periods are 2.0 days (left), 2.5 days (middle), and 3.0 days (right). The top to bottom panels correspond to models (1) to (5), and the solid and dotted lines represent the results with $Z=0.01$ and $Z=0.004$, respectively. The open squares correspond to the onset of RLOF. The model parameters are as follows. a: $M_{2, \mathrm{i}}=8.02 M_{\odot}$; b: $M_{2, \mathrm{i}}=9.62 M_{\odot}, \alpha=0.25 ; \mathrm{c}: M_{2, \mathrm{i}}=9.62 M_{\odot}, \beta=0.25$; $\mathrm{d}: M_{2, \mathrm{i}}=9.62 M_{\odot}, \epsilon=0.25 ; \mathrm{e}_{1}: M_{2, \mathrm{i}}=8.12 M_{\odot}, \delta=0.015 ; \mathrm{e}_{2}: M_{2, \mathrm{i}}=8.12 M_{\odot}, \delta=0.025$ for $Z=0.01$ and $\delta=0.03$ for $Z=0.004 ; \mathrm{e}_{3}: M_{2, \mathrm{i}}=8.12 M_{\odot}, \delta=0.03$ for $Z=0.01$ and $\delta=0.08$ for $Z=0.004$. 

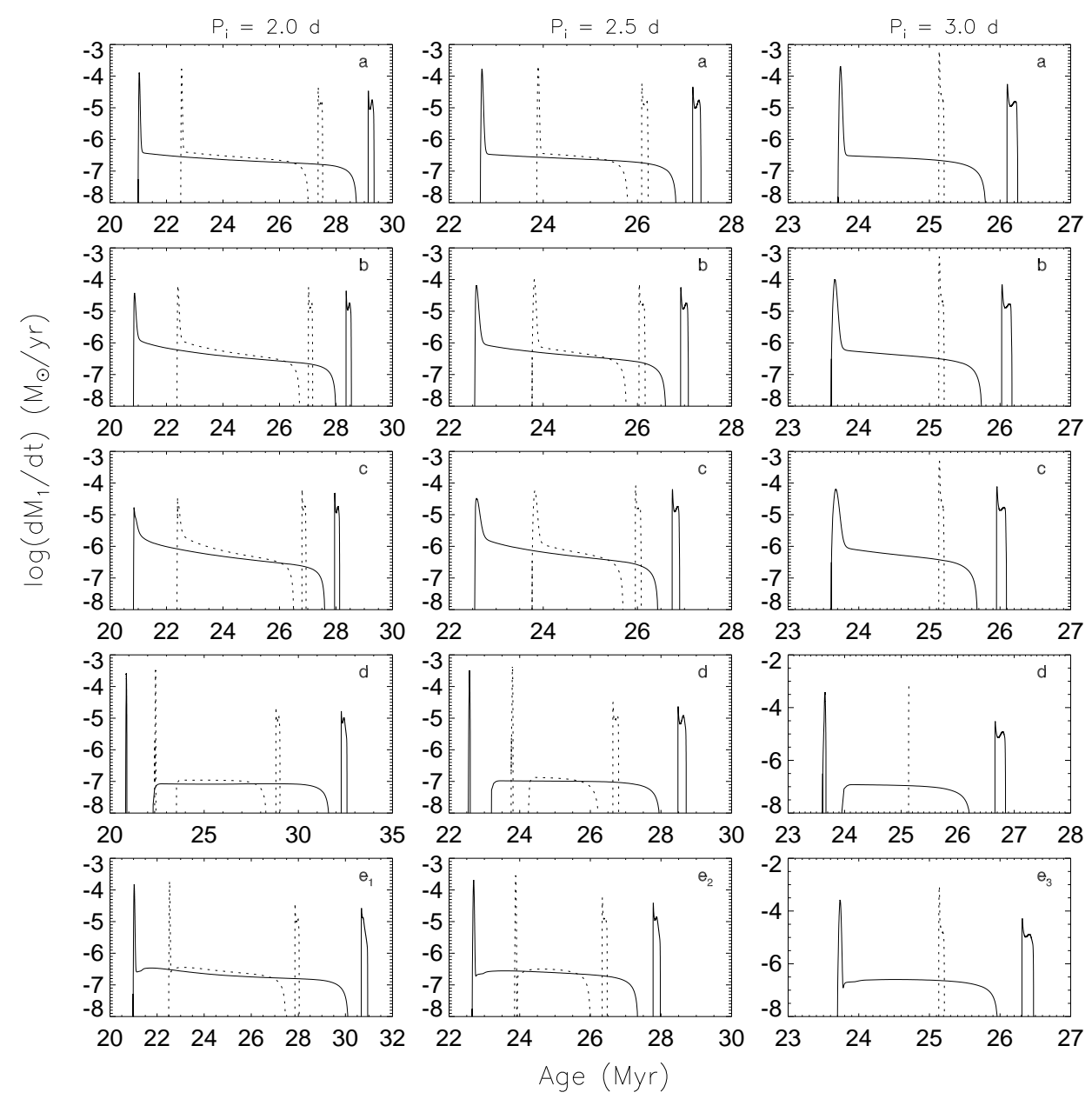

Fig. 3.- Evolution of the mass transfer rate $\dot{M}_{1}$ for different binary models. The initial orbital periods are $P_{\mathrm{i}}=2$ days (left), 3 days (middle), and 4 days (right). Other symbols have the same meanings as in Fig. 2. 


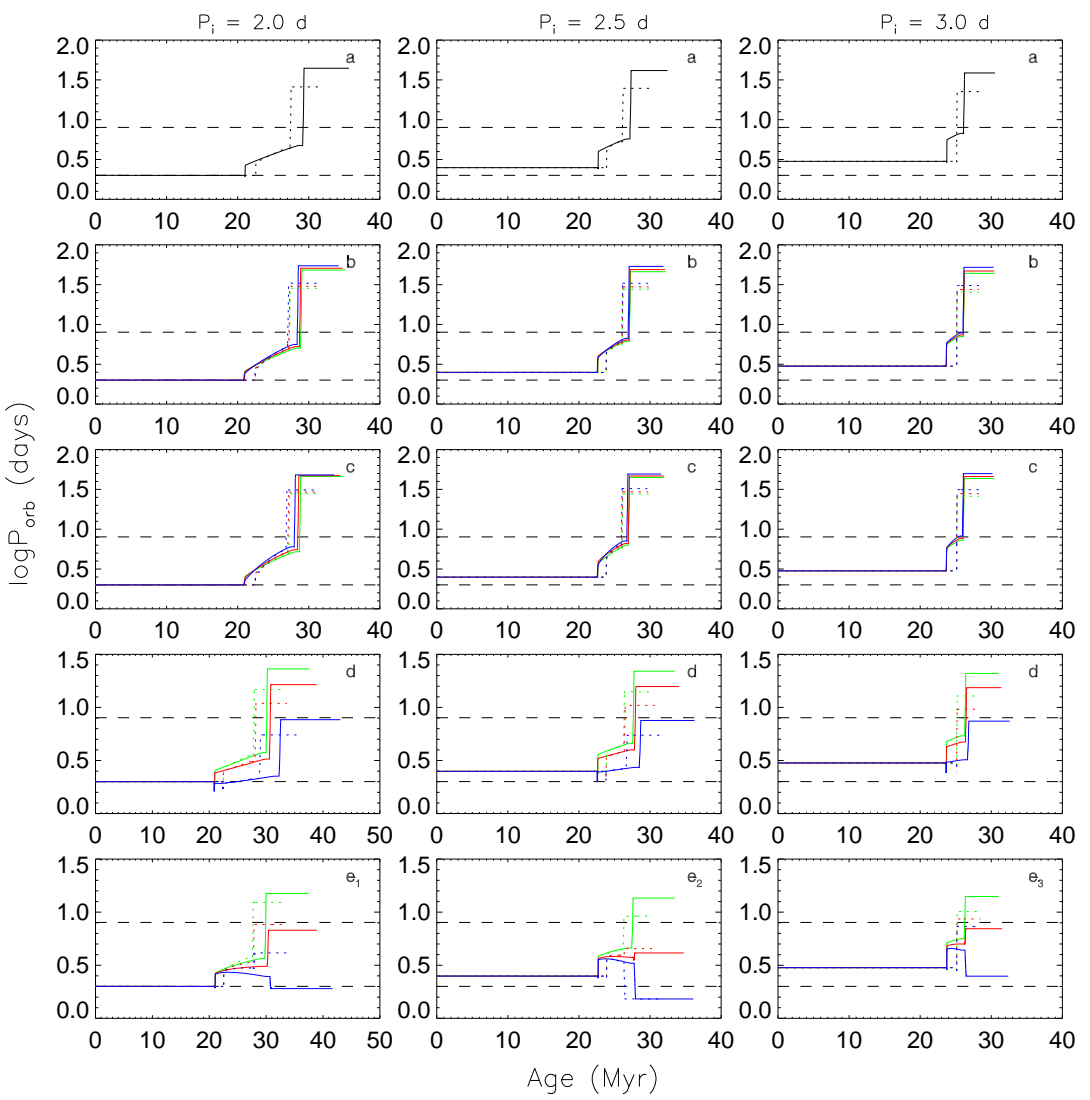

Fig. 4. - Evolution of the orbital period for a ZAMS binary with the primary mass of $9.98 M_{\odot}$. The top to bottom panels correspond to models (1) to (5). The initial orbital periods are $P_{\mathrm{i}}=2$ days (left), 3 days (middle), and 4 days (right), and the solid and dotted lines represent $Z=0.01$ and $Z=0.004$, respectively. Other model parameters are as follows. a: $M_{2, \mathrm{i}}=8.02 M_{\odot} ; \mathrm{b}: M_{2, \mathrm{i}}=8.62 M_{\odot}, \alpha=0.1$ (green), $M_{2, \mathrm{i}}=9.02 M_{\odot}, \alpha=0.15($ red $)$, and $M_{2, \mathrm{i}}=9.62 M_{\odot}, \alpha=0.25$ (blue); c: $M_{2, \mathrm{i}}=8.62 M_{\odot}, \beta=0.1$ (green), $M_{2, \mathrm{i}}=9.02 M_{\odot}$, $\beta=0.15($ red $)$, and $M_{2, \mathrm{i}}=9.62 M_{\odot}, \beta=0.25$ (blue); d: $M_{2, \mathrm{i}}=8.62 M_{\odot}, \epsilon=0.1$ (green), $M_{2, \mathrm{i}}=9.02 M_{\odot}, \epsilon=0.15(\mathrm{red})$, and $M_{2, \mathrm{i}}=9.62 M_{\odot}, \epsilon=0.25$ (blue); $\mathrm{e}_{1}: M_{2, \mathrm{i}}=8.12 M_{\odot}$, $\delta=0.01$ (green), 0.015 (red) and 0.02 (blue); $\mathrm{e}_{2}: M_{2, \mathrm{i}}=8.12 M_{\odot}, \delta=0.015$ (green), 0.025 (red), 0.03 (blue) for $Z=0.01$ and $\delta=0.02$ (green), 0.03 (red), 0.04 (blue) for $Z=0.004$; $\mathrm{e}_{3}$ : $M_{2, \mathrm{i}}=8.12 M_{\odot}, \delta=0.04$ (green), 0.05 (red), 0.06 (blue) for $Z=0.01$ and $\delta=0.06$ (green), 0.07 (red), 0.08 (blue) for $Z=0.004$. The dashed horizontal lines indicate the approximate range of the pre-SN period that can be expected to lead to the formation of SMC X-1. 


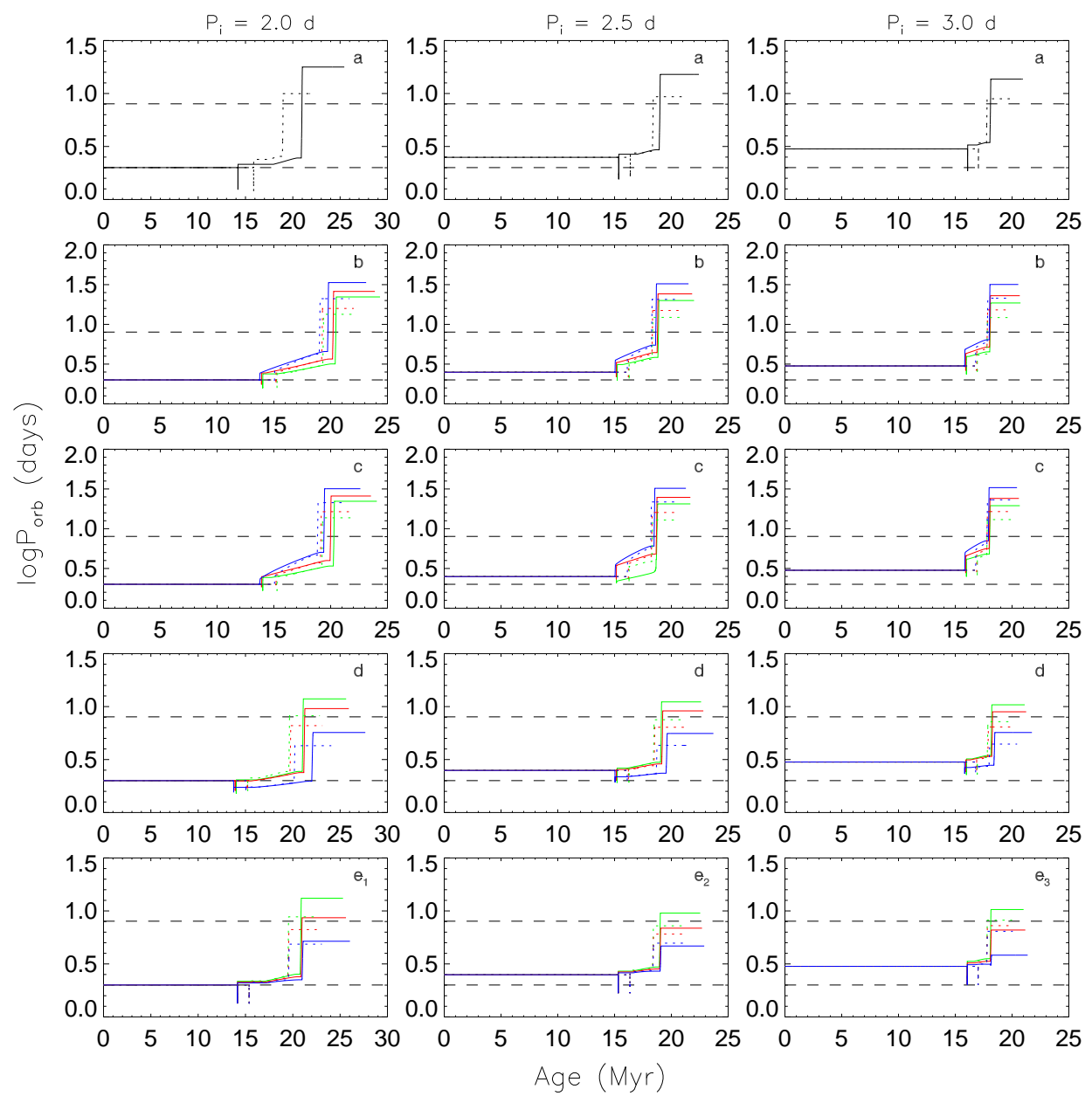

Fig. 5.- Same as Fig. 4, but for a ZAMS binary with the primary mass of $12.56 M_{\odot}$. The model parameters are as follows. a: $M_{2, \mathrm{i}}=5.60 M_{\odot}$; b-d: $M_{2, \mathrm{i}}=7.00 M_{\odot}, \alpha=\beta=\epsilon=0.1$ (green), $M_{2, \mathrm{i}}=8.00 M_{\odot}, \alpha=\beta=\epsilon=0.15$ (red), and $M_{2, \mathrm{i}}=10.00 M_{\odot}, \alpha=\beta=\epsilon=0.25$ (blue); $\mathrm{e}_{1}: M_{2, \mathrm{i}}=6.00 M_{\odot}, \delta=0.005$ (green), 0.01 (red) and 0.015 (blue); $\mathrm{e}_{2}: M_{2, \mathrm{i}}=6.00 M_{\odot}$, $\delta=0.01$ (green), 0.015 (red) and 0.02 (blue); $\mathrm{e}_{3}: M_{2, \mathrm{i}}=6.00 M_{\odot}, \delta=0.01$ (green), 0.02 (red), 0.03 (blue) for $Z=0.01$ and $\delta=0.015$ (green), 0.025 (red), 0.035 (blue) for $Z=0.004$. 
Table 1. Calculated Results of the Binary Evolution with $M_{1, \mathrm{i}}=9.98 M_{\odot}$ in models (1)-(4).

\begin{tabular}{|c|c|c|c|c|c|c|c|c|c|c|c|}
\hline$P_{\text {orb }, \mathrm{i}}($ days $)$ & $M_{2, \mathrm{i}}\left(M_{\odot}\right)$ & $\begin{array}{c}\text { mass loss fraction } \\
\quad \alpha, \beta, \gamma \text {, or } \epsilon\end{array}$ & Model & Case & $M_{1, \mathrm{f}}\left(M_{\odot}\right)$ & $\begin{array}{c}Z=0.01 \\
M_{2, \mathrm{f}}\left(M_{\odot}\right)\end{array}$ & $P_{\text {orb }, \mathrm{f}}($ days $)$ & Case & $M_{1, \mathrm{f}}\left(M_{\odot}\right)$ & $\begin{array}{l}Z=0.004 \\
M_{2, \mathrm{f}}\left(M_{\odot}\right)\end{array}$ & $P_{\text {orb }, \mathrm{f}}$ (days) \\
\hline \multirow[t]{10}{*}{1.5} & 8.02 & 0 & 1 & $\mathrm{~A}$ & 1.53 & 16.47 & 48.38 & $\mathrm{~A}$ & 1.85 & 16.15 & 28.70 \\
\hline & 8.62 & 0.10 & 2 & $\mathrm{~A}$ & 1.57 & 16.19 & 51.42 & $\mathrm{~A}$ & 1.90 & 15.89 & 30.82 \\
\hline & & & 3 & $\mathrm{~A}$ & 1.59 & 16.17 & 47.63 & $\mathrm{~A}$ & 1.92 & 15.87 & 29.40 \\
\hline & & & 4 & $\mathrm{~A}$ & 1.38 & 16.43 & 24.93 & $\mathrm{~A}$ & 1.69 & 16.16 & 15.94 \\
\hline & 9.02 & 0.15 & 2 & $\mathrm{~A}$ & 1.60 & 16.14 & 53.29 & $\mathrm{~A}$ & 1.93 & 15.86 & 32.11 \\
\hline & & & 3 & $\mathrm{~A}$ & 1.63 & 16.12 & 47.55 & $\mathrm{~A}$ & 1.96 & 15.84 & 29.86 \\
\hline & & & 4 & $\mathrm{~A}$ & 1.29 & 16.48 & 17.68 & $\mathrm{~A}$ & 1.59 & 16.22 & 11.64 \\
\hline & 9.62 & 0.25 & 2 & $\mathrm{~A}$ & 1.65 & 15.87 & 56.23 & $\mathrm{~A}$ & 1.98 & 15.62 & 34.06 \\
\hline & & & 3 & $\mathrm{~A}$ & 1.69 & 15.83 & 47.02 & $\mathrm{~A}$ & 2.03 & 15.58 & 30.33 \\
\hline & & & 4 & $\mathrm{~A}$ & 1.07 & 16.39 & 8.50 & $\mathrm{~A}$ & 1.36 & 16.16 & 5.82 \\
\hline \multirow[t]{10}{*}{2.0} & 8.02 & 0 & 1 & $\mathrm{~A}$ & 1.75 & 16.25 & 44.30 & $\mathrm{~A}$ & 2.15 & 15.85 & 25.82 \\
\hline & 8.62 & 0.10 & 2 & $\mathrm{~A}$ & 1.79 & 15.99 & 48.25 & A & 2.19 & 15.64 & 28.48 \\
\hline & & & 3 & $\mathrm{~A}$ & 1.81 & 15.98 & 45.95 & $\mathrm{~A}$ & 2.20 & 15.63 & 27.98 \\
\hline & & & 4 & $\mathrm{~A}$ & 1.63 & 16.21 & 23.06 & $\mathrm{~A}$ & 2.00 & 15.87 & 14.74 \\
\hline & 9.02 & 0.15 & 2 & $\mathrm{~A}$ & 1.81 & 15.96 & 50.81 & $\mathrm{~A}$ & 2.21 & 15.63 & 30.17 \\
\hline & & & 3 & $\mathrm{~A}$ & 1.84 & 15.94 & 47.06 & A & 2.22 & 15.61 & 29.29 \\
\hline & & & 4 & $\mathrm{~A}$ & 1.55 & 16.26 & 16.38 & A & 1.91 & 15.95 & 10.92 \\
\hline & 9.62 & 0.25 & 2 & $\mathrm{~A}$ & 1.85 & 15.72 & 54.55 & $\mathrm{~A}$ & 2.24 & 15.43 & 32.93 \\
\hline & & & 3 & $\mathrm{~A}$ & 1.89 & 15.69 & 48.04 & A & 2.24 & 15.43 & 32.19 \\
\hline & & & 4 & A & 1.36 & 16.14 & 7.66 & A & 1.69 & 15.88 & 5.51 \\
\hline \multirow[t]{10}{*}{2.5} & 8.02 & 0 & 1 & A & 1.96 & 16.04 & 41.43 & A & 2.39 & 15.61 & 24.77 \\
\hline & 8.62 & 0.10 & 2 & $\mathrm{~A}$ & 1.98 & 15.82 & 45.94 & A & 2.41 & 15.43 & 27.71 \\
\hline & & & 3 & $\mathrm{~A}$ & 1.99 & 15.81 & 44.63 & $\mathrm{~A}$ & 2.41 & 15.43 & 27.67 \\
\hline & & & 4 & $\mathrm{~A}$ & 1.84 & 16.01 & 21.94 & $\mathrm{~A}$ & 2.28 & 15.62 & 14.02 \\
\hline & 9.02 & 0.15 & 2 & $\mathrm{~A}$ & 2.00 & 15.80 & 48.84 & $\mathrm{~A}$ & 2.42 & 15.44 & 29.64 \\
\hline & & & 3 & $\mathrm{~A}$ & 2.02 & 15.79 & 46.61 & $\mathrm{~A}$ & 2.43 & 15.44 & 29.47 \\
\hline & & & 4 & A & 1.78 & 16.07 & 15.72 & $\mathrm{~A}$ & 2.18 & 15.73 & 10.71 \\
\hline & 9.62 & 0.25 & 2 & $\mathrm{~A}$ & 2.03 & 15.58 & 53.53 & A & 2.44 & 15.27 & 32.86 \\
\hline & & & 3 & A & 2.06 & 15.56 & 49.25 & A & 2.46 & 15.26 & 32.34 \\
\hline & & & 4 & A & 1.59 & 15.96 & 7.53 & A & 2.01 & 15.73 & 5.47 \\
\hline \multirow[t]{2}{*}{3.0} & 8.02 & 0 & 1 & A & 2.15 & 15.85 & 38.63 & B & 2.63 & 15.37 & 23.27 \\
\hline & 8.62 & 0.10 & 2 & A & 2.16 & 15.65 & 43.83 & B & 2.67 & 15.20 & 25.54 \\
\hline
\end{tabular}


Table 1-Continued

\begin{tabular}{|c|c|c|c|c|c|c|c|c|c|c|c|}
\hline$P_{\text {orb }, \mathrm{i}}($ days $)$ & $M_{2, \mathrm{i}}\left(M_{\odot}\right)$ & $\begin{array}{l}\text { mass loss fraction } \\
\qquad \alpha, \beta, \gamma \text {, or } \epsilon\end{array}$ & Model & Case & $M_{1, \mathrm{f}}\left(M_{\odot}\right)$ & $\begin{array}{c}Z=0.01 \\
M_{2, \mathrm{f}}\left(M_{\odot}\right)\end{array}$ & $P_{\text {orb,f }}($ days $)$ & Case & $M_{1, \mathrm{f}}\left(M_{\odot}\right)$ & $\begin{array}{l}Z=0.004 \\
M_{2, \mathrm{f}}\left(M_{\odot}\right)\end{array}$ & $P_{\text {orb }, \mathrm{f}}$ (days) \\
\hline & & & 3 & A & 2.17 & 15.65 & 43.33 & B & 2.67 & 15.20 & 25.89 \\
\hline & & & 4 & A & 2.05 & 15.82 & 20.90 & B & 2.61 & 15.32 & 12.83 \\
\hline & 9.02 & 0.15 & 2 & A & 2.18 & 15.65 & 46.92 & B & 2.68 & 15.23 & 27.50 \\
\hline & & & 3 & A & 2.18 & 15.65 & 45.92 & B & 2.68 & 15.22 & 27.94 \\
\hline & & & 4 & $\mathrm{~A}$ & 1.99 & 15.90 & 15.34 & B & 2.53 & 15.43 & 9.84 \\
\hline & 9.62 & 0.25 & 2 & A & 2.19 & 15.46 & 52.20 & B & 2.69 & 15.09 & 30.82 \\
\hline & & & 3 & $\mathrm{~A}$ & 2.21 & 15.45 & 49.82 & B & 2.69 & 15.09 & 31.39 \\
\hline & & & 4 & A & 1.83 & 15.79 & 7.42 & B & & $\dot{M}$ divergent & \\
\hline \multirow{10}{*}{3.5} & 8.02 & 0 & 1 & A & 2.36 & 15.64 & 35.83 & B & 2.68 & 15.32 & 26.00 \\
\hline & 8.62 & 0.10 & 2 & A & 2.36 & 15.48 & 41.01 & B & 2.69 & 15.18 & 29.44 \\
\hline & & & 3 & A & 2.36 & 15.48 & 40.94 & B & 2.69 & 15.18 & 29.86 \\
\hline & & & 4 & $\mathrm{~A}$ & 2.28 & 15.63 & 19.70 & B & 2.63 & 15.30 & 14.73 \\
\hline & 9.02 & 0.15 & 2 & A & 2.36 & 15.50 & 44.28 & B & 2.69 & 15.22 & 31.71 \\
\hline & & & 3 & A & 2.37 & 15.49 & 43.99 & B & 2.69 & 15.21 & 32.24 \\
\hline & & & 4 & A & 2.23 & 15.68 & 14.33 & B & 2.59 & 15.38 & 11.01 \\
\hline & 9.62 & 0.25 & 2 & A & 2.36 & 15.33 & 49.63 & B & 2.70 & 15.08 & 35.55 \\
\hline & & & 3 & A & 2.37 & 15.33 & 49.11 & B & 2.70 & 15.08 & 36.24 \\
\hline & & & 4 & A & 2.10 & 15.51 & 7.19 & B & & $\dot{M}$ divergent & \\
\hline \multirow[t]{10}{*}{4.0} & 8.02 & 0 & 1 & B & 2.52 & 15.48 & 34.49 & B & 2.69 & 15.31 & 29.41 \\
\hline & 8.62 & 0.10 & 2 & B & 2.53 & 15.33 & 39.16 & B & 2.70 & 15.17 & 33.32 \\
\hline & & & 3 & B & 2.53 & 15.33 & 39.51 & B & 2.70 & 15.17 & 33.80 \\
\hline & & & 4 & B & 2.47 & 15.44 & 18.99 & B & 2.64 & 15.29 & 16.67 \\
\hline & 9.02 & 0.15 & 2 & B & 2.53 & 15.35 & 42.26 & B & 2.70 & 15.21 & 35.89 \\
\hline & & & 3 & B & 2.53 & 15.35 & 42.63 & B & 2.70 & 15.20 & 36.50 \\
\hline & & & 4 & B & 2.44 & 15.50 & 13.97 & B & 2.61 & 15.36 & 12.47 \\
\hline & 9.62 & 0.25 & 2 & B & 2.54 & 15.20 & 47.52 & $\mathrm{~B}$ & 2.71 & 15.07 & 40.24 \\
\hline & & & 3 & B & 2.54 & 15.20 & 47.81 & B & 2.71 & 15.07 & 41.06 \\
\hline & & & 4 & B & & $\dot{M}$ divergent & & B & & $\dot{M}$ divergent & \\
\hline 5.0 & 8.02 & 0 & 1 & B & 2.54 & 15.46 & 42.47 & B & 2.71 & 15.29 & 36.16 \\
\hline 7.0 & 8.02 & 0 & 1 & $\mathrm{~B}$ & 2.56 & 15.44 & 58.20 & B & 2.73 & 15.27 & 49.43 \\
\hline 10.0 & 8.02 & 0 & 1 & B & 2.58 & 15.42 & 81.51 & B & 2.76 & 15.24 & 69.03 \\
\hline
\end{tabular}


Table 2. Calculated Results of the Binary Evolution in model (5) with $M_{1, \mathrm{i}}=9.98 M_{\odot}$, $M_{2, \mathrm{i}}=8.12 M_{\odot}$ and $Z=0.01$.

\begin{tabular}{|c|c|c|c|c|c|}
\hline$P_{\text {orb }, \mathrm{i}}($ days $)$ & Model & mass loss fraction $\delta$ & $M_{1, \mathrm{f}}\left(M_{\odot}\right)$ & $M_{2, \mathrm{f}}\left(M_{\odot}\right)$ & $P_{\text {orb }, \mathrm{f}}($ days $)$ \\
\hline \multirow[t]{3}{*}{2.0} & 5 & 0.01 & 1.61 & 16.42 & 15.02 \\
\hline & & 0.015 & 1.51 & 16.48 & 6.76 \\
\hline & & 0.02 & 1.34 & 16.60 & 1.91 \\
\hline \multirow[t]{4}{*}{2.5} & 5 & 0.015 & 1.81 & 16.18 & 13.58 \\
\hline & & 0.02 & 1.75 & 16.21 & 8.06 \\
\hline & & 0.025 & 1.66 & 16.26 & 4.13 \\
\hline & & 0.03 & 1.53 & 16.34 & 1.52 \\
\hline \multirow[t]{5}{*}{3.0} & 5 & 0.02 & 2.03 & 15.93 & 14.03 \\
\hline & & 0.025 & 1.99 & 15.93 & 10.15 \\
\hline & & 0.03 & 1.94 & 15.95 & 6.98 \\
\hline & & 0.035 & 1.87 & 15.98 & 4.48 \\
\hline & & 0.04 & 1.79 & 16.02 & 2.49 \\
\hline \multirow[t]{5}{*}{3.5} & 5 & 0.03 & 2.27 & 15.63 & 13.55 \\
\hline & & 0.04 & 2.23 & 15.60 & 10.06 \\
\hline & & 0.05 & 2.18 & 15.58 & 6.70 \\
\hline & & 0.06 & 2.12 & 15.57 & 4.20 \\
\hline & & 0.07 & 2.04 & 15.58 & 2.42 \\
\hline
\end{tabular}


Table 3. Calculated Results of the Binary Evolution in model (5) with $M_{1, \mathrm{i}}=9.98 M_{\odot}$, $M_{2, \mathrm{i}}=8.12 M_{\odot}$ and $Z=0.004$.

\begin{tabular}{|c|c|c|c|c|c|}
\hline$P_{\text {orb }, \mathrm{i}}($ days $)$ & Model & mass loss fraction $\delta$ & $M_{1, \mathrm{f}}\left(M_{\odot}\right)$ & $M_{2, \mathrm{f}}\left(M_{\odot}\right)$ & $P_{\text {orb }, \mathrm{f}}$ (days) \\
\hline \multirow[t]{5}{*}{2.0} & 5 & 0.005 & 2.09 & 15.98 & 18.44 \\
\hline & & 0.01 & 2.01 & 16.02 & 12.36 \\
\hline & & 0.015 & 1.92 & 16.08 & 7.66 \\
\hline & & 0.02 & 1.80 & 16.15 & 4.13 \\
\hline & & 0.025 & 1.64 & 16.27 & 1.88 \\
\hline \multirow[t]{4}{*}{2.5} & 5 & 0.01 & 2.32 & 15.72 & 15.85 \\
\hline & & 0.02 & 2.23 & 15.74 & 9.17 \\
\hline & & 0.03 & 2.10 & 15.79 & 4.54 \\
\hline & & 0.04 & 1.89 & 15.92 & 1.52 \\
\hline \multirow[t]{6}{*}{3.0} & 5 & 0.04 & 2.62 & 15.22 & 13.57 \\
\hline & & 0.05 & 2.60 & 15.18 & 11.77 \\
\hline & & 0.06 & 2.58 & 15.14 & 10.14 \\
\hline & & 0.07 & 2.55 & 15.09 & 8.64 \\
\hline & & 0.08 & 2.54 & 15.04 & 7.31 \\
\hline & & 0.09 & & $\dot{M}$ divergent & \\
\hline
\end{tabular}


Table 4. Calculated Results of the Binary Evolution with $M_{1, \mathrm{i}}=12.56 M_{\odot}$ in models (1)-(4).

\begin{tabular}{|c|c|c|c|c|c|c|c|c|c|c|c|}
\hline$P_{\text {orb }, \mathrm{i}}($ days $)$ & $M_{2, \mathrm{i}}\left(M_{\odot}\right)$ & $\begin{array}{c}\text { mass loss fraction } \\
\alpha, \beta, \gamma, \text { or } \epsilon\end{array}$ & Model & Case & $M_{1, \mathrm{f}}\left(M_{\odot}\right)$ & $\begin{array}{c}Z=0.01 \\
M_{2, \mathrm{f}}\left(M_{\odot}\right)\end{array}$ & $P_{\text {orb }, \mathrm{f}}($ days $)$ & Case & $M_{1, \mathrm{f}}\left(M_{\odot}\right)$ & $\begin{array}{l}Z=0.004 \\
M_{2, \mathrm{f}}\left(M_{\odot}\right)\end{array}$ & $P_{\text {orb }, \mathrm{f}}$ (days) \\
\hline \multirow[t]{13}{*}{2.0} & 5.60 & 0 & 1 & $\mathrm{~A}$ & 2.11 & 16.05 & 17.79 & $\mathrm{~A}$ & 2.70 & 15.46 & 9.98 \\
\hline & 7.00 & 0.10 & 2 & $\mathrm{~A}$ & 2.25 & 16.28 & 23.14 & $\mathrm{~A}$ & 2.79 & 15.79 & 13.38 \\
\hline & & & 3 & $\mathrm{~A}$ & 2.33 & 16.21 & 22.14 & $\mathrm{~A}$ & 2.83 & 15.75 & 13.72 \\
\hline & & & 4 & $\mathrm{~A}$ & 2.09 & 16.71 & 11.82 & $\mathrm{~A}$ & 2.56 & 16.26 & 7.50 \\
\hline & 8.00 & 0.15 & 2 & $\mathrm{~A}$ & 2.37 & 16.66 & 25.97 & $\mathrm{~A}$ & 2.88 & 16.23 & 15.85 \\
\hline & & & 3 & $\mathrm{~A}$ & 2.42 & 16.62 & 25.81 & $\mathrm{~A}$ & 2.92 & 16.19 & 16.37 \\
\hline & & & 4 & $\mathrm{~A}$ & 2.05 & 17.27 & 9.57 & $\mathrm{~A}$ & 2.51 & 16.88 & 6.61 \\
\hline & 10.0 & 0.25 & 2 & $\mathrm{~A}$ & 2.51 & 17.54 & 33.54 & $\mathrm{~A}$ & 3.00 & 17.17 & 21.06 \\
\hline & & & 3 & $\mathrm{~A}$ & 2.59 & 17.48 & 31.86 & $\mathrm{~A}$ & 3.07 & 17.11 & 21.26 \\
\hline & & & 4 & $\mathrm{~A}$ & 1.87 & 18.36 & 5.69 & $\mathrm{~A}$ & 2.30 & 18.10 & 4.20 \\
\hline & 11.50 & 0.50 & 2 & $\mathrm{~A}$ & 2.64 & 16.46 & 39.75 & $\mathrm{~A}$ & 3.13 & 16.21 & 25.43 \\
\hline & & & 3 & $\mathrm{~A}$ & 2.80 & 16.38 & 36.58 & $\mathrm{~A}$ & 3.29 & 16.14 & 25.72 \\
\hline & & & 4 & $\mathrm{~A}$ & & $\dot{M}$ divergent & & $\mathrm{A}$ & & $\dot{M}$ divergent & \\
\hline \multirow[t]{13}{*}{2.5} & 5.60 & 0 & 1 & $\mathrm{~A}$ & 2.46 & 15.70 & 15.14 & $\mathrm{~A}$ & 2.93 & 15.23 & 9.34 \\
\hline & 7.00 & 0.10 & 2 & $\mathrm{~A}$ & 2.60 & 15.96 & 19.94 & $\mathrm{~A}$ & 3.17 & 15.45 & 12.24 \\
\hline & & & 3 & $\mathrm{~A}$ & 2.63 & 15.93 & 20.52 & $\mathrm{~A}$ & 3.20 & 15.43 & 12.89 \\
\hline & & & 4 & $\mathrm{~A}$ & 2.41 & 16.41 & 10.90 & $\mathrm{~A}$ & 2.95 & 15.92 & 7.20 \\
\hline & 8.00 & 0.15 & 2 & $\mathrm{~A}$ & 2.66 & 16.42 & 24.20 & $\mathrm{~A}$ & 3.22 & 15.94 & 14.96 \\
\hline & & & 3 & $\mathrm{~A}$ & 2.70 & 16.38 & 24.82 & $\mathrm{~A}$ & 3.25 & 15.92 & 16.01 \\
\hline & & & 4 & $\mathrm{~A}$ & 2.38 & 17.00 & 9.11 & $\mathrm{~A}$ & 2.88 & 16.57 & 6.51 \\
\hline & 10.00 & 0.25 & 2 & $\mathrm{~A}$ & 2.77 & 17.34 & 32.39 & $\mathrm{~A}$ & 3.31 & 16.93 & 20.61 \\
\hline & & & 3 & $\mathrm{~A}$ & 2.83 & 17.30 & 32.27 & $\mathrm{~A}$ & 3.35 & 16.90 & 21.73 \\
\hline & & & 4 & $\mathrm{~A}$ & 2.20 & 18.90 & 5.58 & $\mathrm{~A}$ & 2.72 & 17.73 & 4.31 \\
\hline & 11.50 & 0.50 & 2 & $\mathrm{~A}$ & 2.87 & 16.34 & 39.90 & $\mathrm{~A}$ & 3.39 & 16.09 & 25.94 \\
\hline & & & 3 & $\mathrm{~A}$ & 2.99 & 16.28 & 39.39 & $\mathrm{~A}$ & 3.48 & 16.04 & 28.42 \\
\hline & & & 4 & $\mathrm{~A}$ & & $\dot{M}$ divergent & & $\mathrm{A}$ & & $\dot{M}$ divergent & \\
\hline \multirow[t]{6}{*}{3.0} & 5.60 & 0 & 1 & A & 2.75 & 15.41 & 13.67 & $\mathrm{~A}$ & 3.23 & 14.93 & 8.93 \\
\hline & 7.00 & 0.10 & 2 & $\mathrm{~A}$ & 2.88 & 15.71 & 18.60 & $\mathrm{~A}$ & 3.43 & 15.22 & 12.19 \\
\hline & & & 3 & $\mathrm{~A}$ & 2.90 & 15.69 & 19.50 & $\mathrm{~A}$ & 3.44 & 15.20 & 13.02 \\
\hline & & & 4 & $\mathrm{~A}$ & 2.71 & 16.15 & 10.39 & $\mathrm{~A}$ & 3.28 & 15.63 & 7.21 \\
\hline & 8.00 & 0.15 & 2 & $\mathrm{~A}$ & 2.92 & 16.20 & 22.93 & $\mathrm{~A}$ & 3.45 & 15.74 & 15.19 \\
\hline & & & 3 & $\mathrm{~A}$ & 2.95 & 16.17 & 24.07 & $\mathrm{~A}$ & 3.47 & 15.72 & 16.43 \\
\hline
\end{tabular}


Table 4-Continued

\begin{tabular}{|c|c|c|c|c|c|c|c|c|c|c|c|}
\hline$P_{\text {orb }, \mathrm{i}}$ (days) & $M_{2, \mathrm{i}}\left(M_{\odot}\right)$ & $\begin{array}{c}\text { mass loss fraction } \\
\alpha, \beta, \gamma, \text { or } \epsilon\end{array}$ & Model & Case & $M_{1, \mathrm{f}}\left(M_{\odot}\right)$ & $\begin{array}{c}Z=0.01 \\
M_{2, \mathrm{f}}\left(M_{\odot}\right)\end{array}$ & $P_{\text {orb }, \mathrm{f}}($ days $)$ & Case & $M_{1, \mathrm{f}}\left(M_{\odot}\right)$ & $\begin{array}{l}Z=0.004 \\
M_{2, \mathrm{f}}\left(M_{\odot}\right)\end{array}$ & $P_{\text {orb }, \mathrm{f}}($ days $)$ \\
\hline & & & 4 & $\mathrm{~A}$ & 2.67 & 16.75 & 8.93 & $\mathrm{~A}$ & 3.24 & 16.26 & 6.43 \\
\hline & 10.00 & 0.25 & 2 & $\mathrm{~A}$ & 3.00 & 17.17 & 31.77 & $\mathrm{~A}$ & 3.51 & 16.79 & 21.35 \\
\hline & & & 3 & $\mathrm{~A}$ & 3.03 & 17.15 & 32.91 & $\mathrm{~A}$ & 3.54 & 16.77 & 23.09 \\
\hline & & & 4 & $\mathrm{~A}$ & 2.50 & 17.88 & 5.69 & $\mathrm{~A}$ & 3.07 & 17.46 & 4.43 \\
\hline & 11.50 & 0.50 & 2 & A & 3.05 & 16.25 & 40.53 & A & 3.55 & 16.00 & 27.55 \\
\hline & & & 3 & A & 3.16 & 16.20 & 41.99 & A & 3.62 & 15.97 & 31.20 \\
\hline & & & 4 & $\mathrm{~A}$ & & $\dot{M}$ divergent & & $\mathrm{A}$ & & $\dot{M}$ divergent & \\
\hline \multirow[t]{13}{*}{3.5} & 5.60 & 0 & 1 & $\mathrm{~A}$ & 3.01 & 15.15 & 12.74 & B & 3.48 & 14.68 & 8.81 \\
\hline & 7.00 & 0.10 & 2 & $\mathrm{~A}$ & 3.11 & 15.51 & 17.96 & B & 3.64 & 15.03 & 12.35 \\
\hline & & & 3 & $\mathrm{~A}$ & 3.13 & 15.49 & 18.99 & $\mathrm{~B}$ & 3.66 & 15.01 & 13.25 \\
\hline & & & 4 & $\mathrm{~A}$ & 2.98 & 15.90 & 10.04 & B & & $\dot{M}$ divergent & \\
\hline & 8.00 & 0.15 & 2 & $\mathrm{~A}$ & 3.14 & 16.00 & 22.27 & B & 3.68 & 15.54 & 15.19 \\
\hline & & & 3 & $\mathrm{~A}$ & 3.17 & 15.98 & 23.78 & B & 3.70 & 15.53 & 16.65 \\
\hline & & & 4 & $\mathrm{~A}$ & 2.94 & 16.52 & 8.81 & $\mathrm{~B}$ & & $\dot{M}$ divergent & \\
\hline & 10.00 & 0.25 & 2 & $\mathrm{~A}$ & 3.18 & 17.03 & 31.75 & B & 3.72 & 16.63 & 21.56 \\
\hline & & & 3 & $\mathrm{~A}$ & 3.21 & 17.01 & 33.56 & B & 3.74 & 16.62 & 23.81 \\
\hline & & & 4 & $\mathrm{~A}$ & 2.78 & 17.66 & 5.78 & $\mathrm{~B}$ & & $\dot{M}$ divergent & \\
\hline & 11.50 & 0.25 & 2 & $\mathrm{~A}$ & 3.24 & 16.16 & 40.82 & B & 3.75 & 15.90 & 28.11 \\
\hline & & & 3 & $\mathrm{~A}$ & 3.30 & 16.13 & 44.71 & $\mathrm{~B}$ & 3.77 & 15.89 & 33.43 \\
\hline & & 0.25 & 4 & $\mathrm{~A}$ & & $\dot{M}$ divergent & & $\mathrm{B}$ & & $\dot{M}$ divergent & \\
\hline \multirow{12}{*}{4.0} & 5.60 & 0 & 1 & $\mathrm{~B}$ & 3.41 & 14.75 & 10.91 & $\mathrm{~B}$ & 3.51 & 14.65 & 9.94 \\
\hline & 7.00 & 0.10 & 2 & $\mathrm{~B}$ & 3.46 & 15.19 & 15.87 & $\mathrm{~B}$ & 3.66 & 15.01 & 13.94 \\
\hline & & & 3 & $\mathrm{~B}$ & 3.48 & 15.17 & 16.99 & $\mathrm{~B}$ & 3.68 & 14.99 & 14.94 \\
\hline & & & 4 & $\mathrm{~B}$ & & $\dot{M}$ divergent & & $\mathrm{B}$ & & $\dot{M}$ divergent & \\
\hline & 8.00 & 0.15 & 2 & $\mathrm{~B}$ & 3.51 & 15.70 & 19.52 & $\mathrm{~B}$ & 3.70 & 15.53 & 17.19 \\
\hline & & & 3 & B & 3.52 & 15.69 & 21.30 & $\mathrm{~B}$ & 3.71 & 15.52 & 18.88 \\
\hline & & & 4 & B & & $\dot{M}$ divergent & & B & & $\dot{M}$ divergent & \\
\hline & 10.00 & 0.25 & 2 & $\mathrm{~A}$ & 3.39 & 16.88 & 31.03 & $\mathrm{~B}$ & 3.74 & 16.62 & 24.46 \\
\hline & & & 3 & $\mathrm{~A}$ & 3.41 & 16.86 & 33.43 & B & 3.75 & 16.61 & 27.03 \\
\hline & & & 4 & $\mathrm{~A}$ & 3.11 & 17.42 & 5.78 & $\mathrm{~B}$ & & $\dot{M}$ divergent & \\
\hline & 11.50 & 0.50 & 2 & $\mathrm{~A}$ & 3.41 & 16.07 & 40.85 & $\mathrm{~B}$ & 3.76 & 15.90 & 31.91 \\
\hline & & & 3 & $\mathrm{~A}$ & 3.44 & 19.50 & 46.49 & B & 3.78 & 15.89 & 37.99 \\
\hline
\end{tabular}


Table 4-Continued

\begin{tabular}{|c|c|c|c|c|c|c|c|c|c|c|c|}
\hline$P_{\text {orb }, \mathrm{i}}($ days $)$ & $M_{2, \mathrm{i}}\left(M_{\odot}\right)$ & $\begin{array}{l}\text { mass loss fraction } \\
\quad \alpha, \beta, \gamma \text {, or } \epsilon\end{array}$ & Model & Case & $M_{1, \mathrm{f}}\left(M_{\odot}\right)$ & $\begin{array}{c}Z=0.01 \\
M_{2, \mathrm{f}}\left(M_{\odot}\right)\end{array}$ & $P_{\text {orb }, \mathrm{f}}($ days $)$ & Case & $M_{1, \mathrm{f}}\left(M_{\odot}\right)$ & $\begin{array}{l}Z=0.004 \\
M_{2, \mathrm{f}}\left(M_{\odot}\right)\end{array}$ & $P_{\text {orb }, \mathrm{f}}$ (days) \\
\hline & & & 4 & $\mathrm{~A}$ & & $\dot{M}$ divergent & & B & & $\dot{M}$ divergent & \\
\hline 5.0 & 5.60 & 0 & 1 & B & 3.44 & 14.72 & 13.35 & B & 3.54 & 14.62 & 12.17 \\
\hline 7.0 & 5.60 & 0 & 1 & B & 3.49 & 14.67 & 18.15 & B & 3.59 & 14.57 & 16.55 \\
\hline 10.0 & 5.60 & 0 & 1 & B & 3.53 & 14.63 & 25.24 & $\mathrm{~B}$ & 3.63 & 14.53 & 23.01 \\
\hline
\end{tabular}


Table 5. Calculated Results of the Binary Evolution in model (5) with $M_{1, \mathrm{i}}=12.56 M_{\odot}$, $M_{2, \mathrm{i}}=6.00 M_{\odot}$, and $Z=0.01$.

\begin{tabular}{|c|c|c|c|c|c|}
\hline$P_{\text {orb }, \mathrm{i}}$ (days) & Model & mass loss fraction $\delta$ & $M_{1, \mathrm{f}}\left(M_{\odot}\right)$ & $M_{2, \mathrm{f}}\left(M_{\odot}\right)$ & $P_{\text {orb }, \mathrm{f}}($ days $)$ \\
\hline \multirow[t]{3}{*}{2.0} & 5 & 0.005 & 2.12 & 16.41 & 13.18 \\
\hline & & 0.01 & 2.06 & 16.43 & 8.63 \\
\hline & & 0.015 & 1.98 & 16.47 & 5.17 \\
\hline \multirow[t]{4}{*}{2.5} & 5 & 0.005 & 2.46 & 16.07 & 12.74 \\
\hline & & 0.01 & 2.41 & 16.08 & 9.54 \\
\hline & & 0.015 & 2.35 & 16.10 & 6.88 \\
\hline & & 0.02 & 2.29 & 16.13 & 4.67 \\
\hline \multirow[t]{5}{*}{3.0} & 5 & 0.01 & 2.72 & 15.78 & 10.31 \\
\hline & & 0.015 & 2.68 & 15.78 & 8.32 \\
\hline & & 0.02 & 2.63 & 15.79 & 6.61 \\
\hline & & 0.025 & 2.59 & 15.81 & 5.11 \\
\hline & & 0.03 & 2.53 & 15.83 & 3.82 \\
\hline \multirow[t]{7}{*}{3.5} & 5 & 0.01 & 3.01 & 15.49 & 11.12 \\
\hline & & 0.015 & 2.99 & 15.48 & 9.84 \\
\hline & & 0.02 & 2.97 & 15.47 & 8.67 \\
\hline & & 0.025 & 2.94 & 15.46 & 7.63 \\
\hline & & 0.03 & 2.91 & 15.46 & 6.61 \\
\hline & & 0.035 & 2.88 & 15.45 & 5.76 \\
\hline & & 0.04 & 2.86 & 15.45 & 5.02 \\
\hline
\end{tabular}


Table 6. Calculated Results of the Binary Evolution in model (5) with $M_{1, \mathrm{i}}=12.56 M_{\odot}$, $M_{2, \mathrm{i}}=6.00 M_{\odot}$, and $Z=0.004$.

\begin{tabular}{cccccc}
\hline \hline \multirow{2}{*}{$P_{\text {orb }, \mathrm{i}}($ days $)$} & Model & mass loss fraction $\delta$ & $M_{1, \mathrm{f}}\left(M_{\odot}\right)$ & $M_{2, \mathrm{f}}\left(M_{\odot}\right)$ & $P_{\text {orb }, \mathrm{f}}($ days $)$ \\
& \multirow{2}{*}{2.0} & 0.005 & 2.60 & 15.93 & 8.80 \\
& & 0.01 & 2.54 & 15.96 & 6.67 \\
& & 0.015 & 2.46 & 16.00 & 4.86 \\
2.5 & 5 & 0.01 & 2.97 & 15.53 & 7.23 \\
& & 0.015 & 2.92 & 15.54 & 6.05 \\
& & 0.02 & 2.87 & 15.57 & 4.97 \\
3.0 & 0.025 & 2.81 & 15.59 & 4.02 \\
& & 0.015 & 3.30 & 15.14 & 8.18 \\
& & 0.02 & 3.30 & 15.14 & 7.71 \\
& & 0.025 & 3.28 & 15.13 & 7.27 \\
& & 0.03 & 3.27 & 15.11 & 6.84 \\
& & 0.035 & 3.26 & 15.09 & 6.43 \\
\hline
\end{tabular}

